\title{
Detection of changes in speed and direction of motion: Reaction time analysis
}

\author{
EHTIBAR N. DZHAFAROV \\ University of Illinois at Urbana-Champaign, Champaign, Illinois \\ ROBERT SEKULER \\ Brandeis University, Waltham, Massachusetts, \\ and Boston University, Boston, Massachusetts \\ and \\ JÜRI ALLIK \\ University of Tartu, Tartu, Estonia
}

\begin{abstract}
Observers reacted to the change in the movement of a random-dot field whose initial velocity, $V_{0}$, was constant for a random period and then switched abruptly to another value, $V_{1}$. The two movements, both horizontally oriented, were either in the same direction (speed increments or decrements), or in the opposite direction but equal in speed (direction reversals). One of the two velocities, $V_{0}$ or $V_{1}$, could be zero (motion onset and offset, respectively). In the range of speeds used, $0-16 \mathrm{deg} / \mathrm{sec}$ (dps), the mean reaction time (MRT) for a given value of $V_{0}$ depended on $\left|V_{1}-V_{0}\right|$ only: MRT $\approx r+c\left(V_{0}\right) /\left|V_{1}-V_{0}\right|^{\beta}$, where $\beta=2 / 3, r$ is a velocity-independent component of MRT, and $c\left(V_{0}\right)$ is a parameter whose value is constant for low values of $V_{0}(0-4 \mathrm{dps})$, and increases beginning with some value of $V_{0}$ between 4 and $8 \mathrm{dps}$. These and other data reviewed in the paper are accounted for by a model in which the time-position function of a moving target is encoded by mass activation of a network of Reichardt-type encoders. Motion-onset detection $\left(V_{0}=0\right)$ is achieved by weighted temporal summation of the outputs of this network, the weights assigned to activated encoders being proportional to their squared spatial spans. By means of a "subtractive normalization," the visual system effectively reduces the detection of velocity changes (a change from $V_{0}$ to $V_{1}$ ) to the detection of motion onset (a change from 0 to $V_{1}-V_{0}$ ). Subtractive normalization operates by readjustment of weights: the weights of all encoders are amplified or attenuated depending on their spatial spans, temporal spans, and the initial velocity $V_{0}$. Assignment of weights and weighted temporal summation are thought of as special-purpose computations performed on the dynamic array of activations in the motion-encoding network, without affecting the activations themselves.
\end{abstract}

In this paper, we propose a possible mechanism by which the human visual system detects changes in the frontoparallel movement of a visual target. The term mechanism is understood here as a computational algorithm implemented on a quasi-neuronal network of elementary motion encoders. We propose to treat encoding of visual motion and detection of motion changes as two distinctly different parts of the computational algorithm. We consider motion encoding to be a general-purpose computation that is (relatively) task independent, whose outcome is a dynamic array of elementary activations representing the time-position function of a moving target. In our

This research was supported by a University of Ilinois Research Board grant to E.N.D., by U.S. Air Force Office of Scientific Research Grant AFOSR 89-0243 to R.S., and by a James S. McDonnell Foundation grant. Address correspondence to E. N. Dzhafarov, Department of Psychology, University of Illinois at Urbana-Champaign, 603 E. Daniel St., Champaign, IL 61820. model, the time-position function is represented by pairs of spatial positions occupied by a fixed point on the moving target at different pairs of time moments. The dynamic array of elementary activations contains sufficiently rich information about the encoded motion so that specific questions about the motion can be answered by means of special-purpose (task-specific) computations performed on the outputs of the encoding network. Such questions include, among others, "Has the target moved at all?" and "Has it changed its speed or direction?" In our model, the special-purpose computation involved in the detection of motion changes is a particular form of weighted summation (averaging) of the elementary activations across the network: the weights assigned to different elementary encoders depend on the initial movement whose changes are to be detected. The notion of special-purpose detection computations performed on the array of primary encoders is close, but not identical to, the notion of fastlearning task-specific visual modules recently proposed by Poggio, Fahle, and Edelman (1992). 
We studied movement changes that included the beginning of a movement following a period of rest (motion onset), cessation of a movement (motion offset), and changes in motion direction or speed (direction reversals, speed decrements, and speed increments). The scope of our analysis is restricted to one class of moving patterns, namely, high-contrast (well above contrast threshold) spatially localized luminance perturbations (such as a single dot, a thin line, or a random-dot field) that move rigidly along a single straight line, with no changes occurring in either the pattern itself or its background. The pattern's motion then is uniquely determined by a function relating the unidimensional position $x$ of any point taken on the moving pattern to time: $x=x(t)$. We studied the detection of motion changes by means of the simple reaction time (RT) paradigm: observers responded by pressing a key "as rapidly as possible" as soon as any change in the initial movement (or rest) of a target was detected. Consequently, we were concerned not only with the outcome of the hypothetical visual computations, but also with the time course of these computations.

In our experiments, a target moved with a constant velocity, $V_{0}$, which abruptly changed to another value, $V_{1}$, after a certain period of time that was longer than $1 \mathrm{sec}$. We denote this stimulus paradigm schematically as $V_{0} t \rightarrow V_{1} t$. Either of the two $V$ values may be zero; for example, motion-onset stimulus corresponds to $0 \rightarrow V t$. The detection mechanism proposed, however, is applicable to a broader class of motion changes, also generically referred to as changes in motion velocity. Namely, detectability and detection-time predictions can be derived from our model for changes from a uniform rectilinear motion (or rest), $V_{0} t$, to a movement described by any time-position function, $x(t)$, as shown in Figure 1. For example, a critical part of our discussion of motion-onset
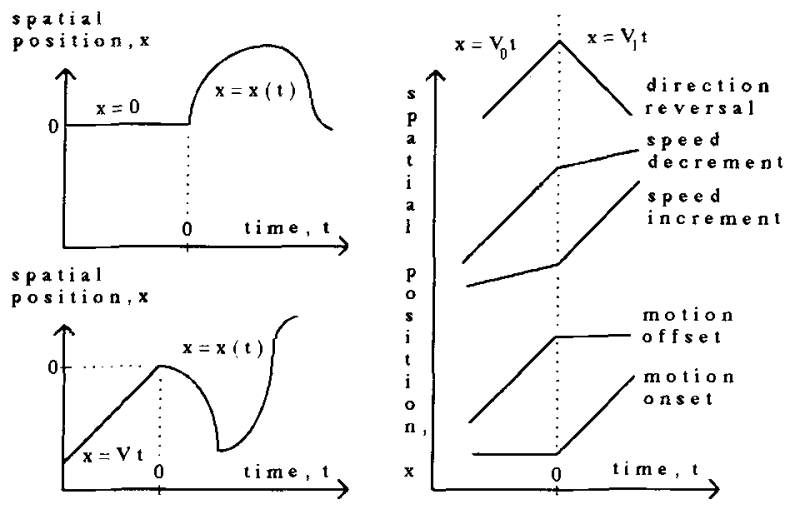

Figure 1. Left panels: Unidimensional spatial position $(x)$ of a target versus time $(t)$ in velocity-change detection (bottom) and, as a special case, in motion-onset detection (top). The period of rest or uniform motion preceding $t=0$ is assumed to last indefinitely. Right panel: The five velocity-change types studied in our experiments: motion onset $\left(V_{0}=0\right)$; motion offset $\left(V_{1}=0\right)$; speed increment $\left(V_{0}<V_{1}\right)$; speed decrement $\left(V_{0}>V_{1}\right)$; and direction reversal $\left(V_{0}=\right.$ $\left.-V_{1}\right)$. detection relates the $0 \rightarrow V_{1} t$ stimulus to the situation in which visual motion is created by a single instantaneous shift in the target position: $0 \rightarrow A$ (a step-function motion of amplitude $A$ ).

Motion-onset detection occupies a special place among other velocity changes: according to the model proposed, detection of a velocity change, $V_{0} t \rightarrow x(t)$, is realized by visual computations of essentially the same structure as detection of the motion onset $0 \rightarrow x(t)-V_{0} t$. In other words, when confronted with the need to detect a change from a uniform motion $V_{0} t$ to another motion, $x(t)$, the detection system effectively subtracts the initial uniform motion from the ongoing motion, $x(t)-V_{0} t$, thereby reducing the task to that of motion-onset detection. In terms of the proposed mechanism, this subtraction is achieved by readjustments of the weights with which the elementary encoding activations are summated.

In velocity-change detection, the existence of an algorithm effectively subtracting the initial uniform motion from the ongoing motion is suggested by the findings of A. B. Sekuler, E. B. Sekuler, and R. Sekuler (1990), who studied reaction times to $30^{\circ}$ changes in direction of motion, with speed held constant. It was shown in their experiments that the mean RT to such changes is not influenced by uncertainty about the initial direction (i.e., by the number and range of possible initial directions), provided that the initial motion lasts longer than about $0.5 \mathrm{sec}$. For shorter durations, the mean RT was shown to increase with uncertainty about the initial direction. It was suggested, therefore, that within the period of about $0.5 \mathrm{sec}$, the motion-processing system learns to subtract the initial motion vector from the ongoing motion, that is, to take the initial vector for a new origin (zero, or "norm of rest") in the space of velocity vectors. Accordingly, this process was termed the normalization (of visual motion in detection of velocity changes); subtractive normalization is more specific, and this is the term we use in this paper.

Assuming that a computational mechanism for motiononset detection is established, one can think of three possible mechanisms for subtractive normalization. The first possibility is the one we elaborate on in this paper: subtractive normalization occurs on the level of specialpurpose computations performed on the activation output of the motion-encoding network. As mentioned earlier, we propose that this is achieved by readjustments of the weights with which elementary activations are summated in the detection computations, without affecting the activations themselves. The second possibility is that subtractive normalization is a sensory adaptation phenomenon, that is, a selective transformation of the activation pattern in the encoding network (e.g., self-inhibition of activated units). We will argue against the sensory adaptation interpretation in the concluding part of our discussion of the normalization mechanisms. The third possibility that one can think of a priori offers a simple mechanistic alternative to computational considerations: detection of the change $V_{0} t \rightarrow x(t)$ is reduced to that of $0 \rightarrow x(t)-V_{0} t$ 
by "aftertracking" eye movements. The assumptions behind this third possibility are (1) that motion-onset-detection computations are performed in retinal rather than external coordinates; (2) that the observers pursue the moving patterns smoothly and accurately (which is not usual, unless they are specifically instructed to pursue; see Hallett, 1986, and Kowler, 1990); and (3) that the initial tracking velocity is maintained for a sufficiently long period after the velocity has changed. Empirical evidence indeed exists (Lightstone, 1973, cited in Hallett, 1986; Mitrani \& Dimitrov, 1978; Mitrani, Dimitrov, Yakimoff, \& Mateeff, 1979; Whittaker \& Eaholtz, 1982) that smooth pursuit continues for several hundred milliseconds after the object being tracked suddenly disappears, stops, or reverses motion direction (the "aftertracking" or "overpursuit" phenomenon).

The simplest test of the aftertracking hypothesis (and of the relevance of the pursuit eye movements in general) is to ask the subject to maintain steady fixation and to monitor the eye movements, discarding all trials when the eyes move. This is precisely what was done in one of the experiments reported in A. B. Sekuler et al. (1990); they found essentially the same course of the normalization process (RT dependence on duration and range of the initial motion directions) under steady fixation as they did under free looking. If aftertracking were the major, or " natural" mechanism for solving the velocity-change-detection task, one would expect that the RT pattern would be disrupted under steady fixation, or at least that the RTs would be lengthened (as the observer would have to relearn the task by different means). The actual data show, if anything, that RTs under steady fixation are somewhat shorter. (One could suggest that the aftertracking hypothesis could be saved by replacing the eye movements with a "moving attentional focus." This interpretation cannot be dismissed by our analysis, except that the computational algorithm we propose can be viewed, if one so wishes, as the mechanism by which the visual system realizes the "moving attentional focus.")

\section{Subtractive Normalization as a Descriptive Term}

We operationally define the concept of subtractive normalization as a particular dependence of RT on velocity changes. As mentioned earlier, the observers in our experiments responded by pressing a key as soon as possible following a detected velocity change, $V_{0} t \rightarrow V_{1} t$. All other stimulus parameters were either held constant or counterbalanced across different values of $V_{0}$ and $V_{1}$ (e.g., luminance/contrast values, duration of the initial phase, spatial pattern of the target, etc.). Consequently, RT can be viewed as determined by the time-position function only, which in turn is determined by the values of $V_{0}$ and $V_{1}: \mathbf{R T}=\mathbf{R T}\left(V_{0}, V_{1}\right)$. (These and subsequent boldface symbols indicate quantities that are random variables.) Consider first the motion-onset-detection task, when $V_{0}=0$. Then, $\mathbf{R T}=\mathbf{R T}(V)$, where $V=V_{1}$. A traditional way to analyze this situation involves two logical steps (see Dzhafarov, 1992, for detailed discussion; see Luce, 1986, for a review).

First, RT as a function of $V$ is additively decomposed into two components, a velocity-dependent component $\mathbf{D}$, and a velocity-independent component $\mathbf{R}$ :

$$
\mathbf{R T}(V)=\mathbf{D}(V)+\mathbf{R},
$$

The velocity-independent component includes not only motor-execution time, but also all sensory and sensorymotor components whose distributions do not change with $V$. The value of $\mathbf{D}$ (stochastically) decreases with increasing $V$; indeed, this is well known to hold for RT as a whole (Ball \& R. Sekuler, 1980; Tynan \& R. Sekuler, 1982), and by definition, $\mathbf{R}$ plays no role in any systematic change of RT as a function of $V$.

Second, some mechanism or computational rule is postulated, whose output determines the detectability level, or "perceptual salience," of motion as a function of $V$. To account for RTs, this output value should be thought of as developing in physical time, continuously or as a step function. The simplest detection rule then defines $\mathbf{D}$ as the period between motion onset and the moment when the developing output value exceeds a certain critical level for the first time. As shown in Dzhafarov (1993), the output can always be considered a deterministic process (depending on $V$ ), whereas the critical value, termed the "criterion," is a random variable, $\mathbf{C}$, whose distribution does not depend on $V$. The component $\mathbf{D}$, therefore, can always be presented as a deterministic function $D(V, \mathbf{C})$ of two arguments: velocity $V$ itself and a $V$-independent random criterion $C$. Equation 1 can now be rewritten as

$$
\mathbf{R T}(V)=D(V, \mathbf{C})+\mathbf{R} \text {. }
$$

Once the function $D(V, \mathrm{C})$ is known, the equation uniquely specifies the dependence of RT on $V$ for any two given values of $\mathbf{C}$ and $\mathbf{R}$. The equation does not, however, specify the distributions of these values, nor does it exclude the possibility that these distributions may change if one changes stimulation parameters other than $V$ (e.g., size of the moving dots, adaptation to a previously presented motion) or task parameters (e.g., speed-accuracy emphasis).

To be less abstract, consider the following model of motion-onset detection (Bonnet, 1977, 1982; Cohen \& Bonnet, 1972; Johnson \& Leibowitz, 1976; for applications to the RT paradigm, see Ball \& R. Sekuler, 1980; Troscianko \& Fahle, 1988; Tynan \& R. Sekuler, 1982). In this so-called critical-distance model, the velocity-dependent component $\mathbf{D}$ is simply the period within which a moving dot traverses some critical distance. Denoting this distance by $\mathbf{C}$, we have the following special case of Equation 2 :

$$
\mathbf{R T}(V)=\mathbf{C}|V|^{-1}+\mathbf{R} \text {. }
$$

For any given values of $\mathbf{C}$ and $\mathbf{R}$, the model uniquely specifies the dependence of RT on $V$ : a power function with the exponent of -1 . Assuming this model, we say that visual motion is subtractively normalized in detection of velocity changes, $V_{0} t \rightarrow V_{1} t$, if 


$$
\mathbf{R T}\left(V_{0}, V_{1}\right)=\mathbf{C}\left|V_{1}-V_{0}\right|^{-1}+\mathbf{R} .
$$

In the general case (Equation 2), one has a subtractive normalization if

$$
\mathbf{R T}\left(V_{0}, V_{1}\right)=D\left(V_{1}-V_{0}, \mathbf{C}\right)+\mathbf{R}
$$

where the distributions of $\mathbf{C}$ and $\mathbf{R}$ may depend on task and stimulation parameters, but not on the value of $V_{1}$. Given that all such parameters are the same for both motion-onset stimuli $\left(V_{0}=0\right)$ and velocity-change stimuli $\left(V_{0}>0\right)$, the only parameter that may systematically influence the distributions of $\mathbf{C}$ and $\mathbf{R}$ is the value of $V_{0}$ (and/or parameters confounded with $V_{0}$, such as the time it takes a single dot to traverse a fixed distance):

$$
\mathbf{C}=\mathbf{C}\left(V_{0}\right) \text { and } \mathbf{R}=\mathbf{R}\left(V_{0}\right) \text {. }
$$

Notice that for detection of motion onset, both $\mathbf{C}$ and $\mathbf{R}$ have been defined as velocity-independent random variables, which for detection of velocity changes translates into their independence from $V_{1}$, but not necessarily from $V_{0}$.

Once the subtractive normalization is found, one can distinguish between its different degrees or forms. For example, assuming that Equation 3 holds, one can speak about a strong subtractive normalization if $\mathbf{R}$ does not depend on $V_{0}$, that is, if its distribution is the same for both motion-onset detection and velocity-change detection. In this case, $\mathbf{R}$ is a "pure delay" with respect to the principal variables of the task; it does not depend on either velocity, $V_{0}$ or $V_{1}$. Recall that $\mathbf{R}$ includes sensory and sensory-motor subcomponents, in addition to motorexecution time. A subtractive normalization, therefore, is not guaranteed to be strong. Finally, if neither $\mathbf{C}$ nor $\mathbf{R}$ exhibit a dependence on $V_{0}$, one can say that subtractive normalization is complete. In this case, the RT distributions are the same for all $\left(V_{0}, V_{1}\right)$ pairs with the same difference $V=V_{1}-V_{0}$.

All these definitions can be trivially generalized to the detection of $V_{0} t \rightarrow x(t)$, where $x(t)$ is not necessarily a uniform motion, $V_{1} t$. We do not do this here, because Equations 1-4 are all we need to analyze our experimental data. In fact, we only use a weak version of these formulas, with RT distributions being characterized by their mean values only. For brevity, however, in most instances we will continue to speak of a subtractive normalization instead of using the more rigorous term "subtractive normalization in the mean."

\section{METHOD}

\section{Procedure}

The display consisted of 200 spatially random, bright dots presented under computer control on a large, dim $x-y$ cathode-ray tube screen (Hewlett Packard Model 1321A, with P-31 phosphor). The background luminance was about $1.5 \mathrm{~cd} / \mathrm{m}^{2}$. The dots were $6^{\prime}$ of arc in diameter, and dot-background contrast was set at four to five times threshold. At the start of each trial, the dots appeared and simultaneously began moving inside a $16^{\circ}$-diam circular aperture. The dots moved horizontally in a fixed spatial phase along parallel paths. When a dot reached the edge of the display, it wrapped around, reap- pearing sometime later at the opposite edge. The dots' velocity was controlled by the size of steps, or displacements, from one frame to the next, keeping frame rate constant at $100 \mathrm{~Hz}$. A new set of spatially random dots was generated on each trial. The display was viewed binocularly with the observer's head steadied in a chinrest. No fixation point was used, and no specifics as to how to view the display were given in the instructions.

The experiment consisted of 35 different conditions, each corresponding to one velocity pair, $\left(V_{0}, V_{1}\right)$. They were tested one at a time in blocks of 50 trials. Over the entire study, each condition was tested on three different occasions, yielding a total of 150 trials per condition. The duration of the $V_{0}$ phase varied according to a uniform distribution ranging from 1 to $2 \mathrm{sec}$. Trials were initiated by the observer.

In 30 conditions, movement during both phases, $V_{0}$ and $V_{1}$, was in a rightward direction. In all these conditions, the subject reacted to a change in speed only. Velocity pairs were chosen from the set of 0 (stationary dots), $1,2,4,8$, and $16 \mathrm{deg} / \mathrm{sec}$ (dps), with the constraint that the two velocities in a condition could not be the same.

In the remaining five conditions, speeds during both phases were the same. In these conditions, rightward motion during the foreperiod changed abruptly to leftward motion, with the same speed. In all these conditions, the subject reacted to a change in direction only. Speeds were $1,2,4,8$, and $16 \mathrm{dps}$.

In addition, we carried out an auxiliary experiment in order to find out whether any of the obtained results could be specifically associated with our choice of the number of dots in the display200. This experiment consisted of 39 different conditions, each corresponding to 1 of 13 velocity pairs $\left(V_{0}, V_{1}\right)$, and one of three dot densities: 50,100 , or 200 dots per screen. A subset of the velocity pairs used in the main experiment was used here: $(0,1),(0,4),(0,16)$; $(1,8) ;(2,1) ;(4,0),(4,16),(4,-4) ;(8,4) ;$ and $(16,0),(16,1),(16,2)$, $(16,-16)$, where the minus sign indicates leftward motion. In all other respects, the auxiliary experiment was identical to the main one.

In both the main and auxiliary experiments, mean RTs were calculated after discarding RTs below $100 \mathrm{msec}$ or greater than $1,000 \mathrm{msec}$. The number of discarded trials was fairly constant for all conditions, and constituted less than $5 \%$ of trials.

\section{Subjects}

One of the observers in the main experiment was an author of this report (R.W.S.); the other observer (J.F.) was naive with respect to the purposes of the study. A third observer (J.L.M.), also naive, served in the auxiliary experiment.

\section{RESULTS}

\section{Ordinal-Level Analysis}

Figure 2 shows the mean RT (MRT) plotted against the values of $V_{1}-V_{0}$. For ease of interpretation, the data for different values of $V_{0}$ are shown in separate panels. The theoretical curves are fitted to the MRTs averaged over Observers J.F. and R.W.S.; the curves are discussed below, but they may be used at this stage as visual aids for determining relative positions of the data points. The curves are shown separately in Figure 3A; the lower curve is the one used to fit the data for $V_{0}=0,1,2$, and $4 \mathrm{dps}$ in Figure 2 (the same curve for all four data sets), the middle and upper curves of Figure $3 \mathrm{~A}$ are used to fit the data for $V_{0}=8$ and $16 \mathrm{dps}$, respectively. Keeping these properties of the curves in mind, one can notice the following characteristics of the data shown in Figure 2.

1. For a given $V_{0}$, MRT decreases as the difference between $V_{1}$ and $V_{0}$ increases in either direction. 


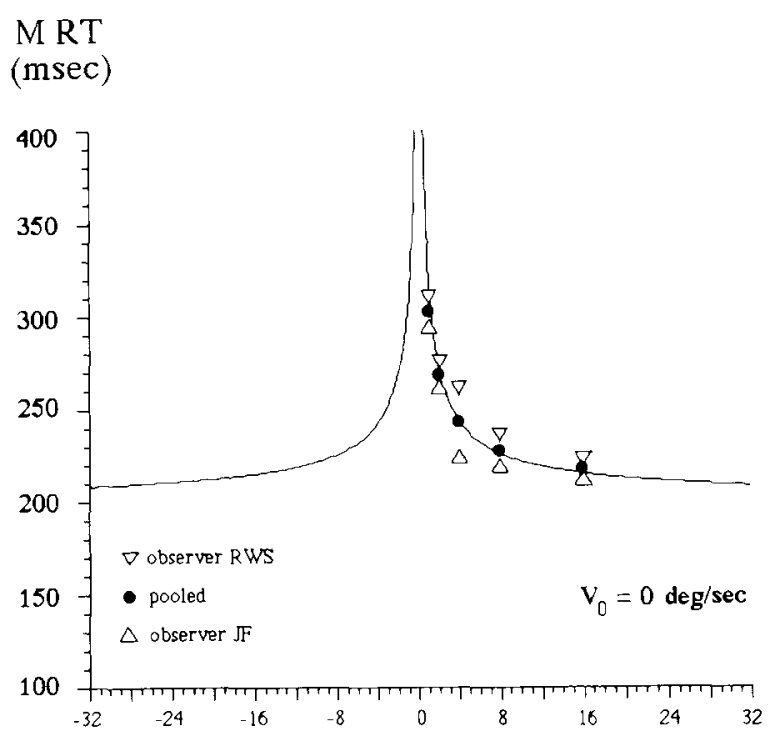

\section{RT \\ (msec)}
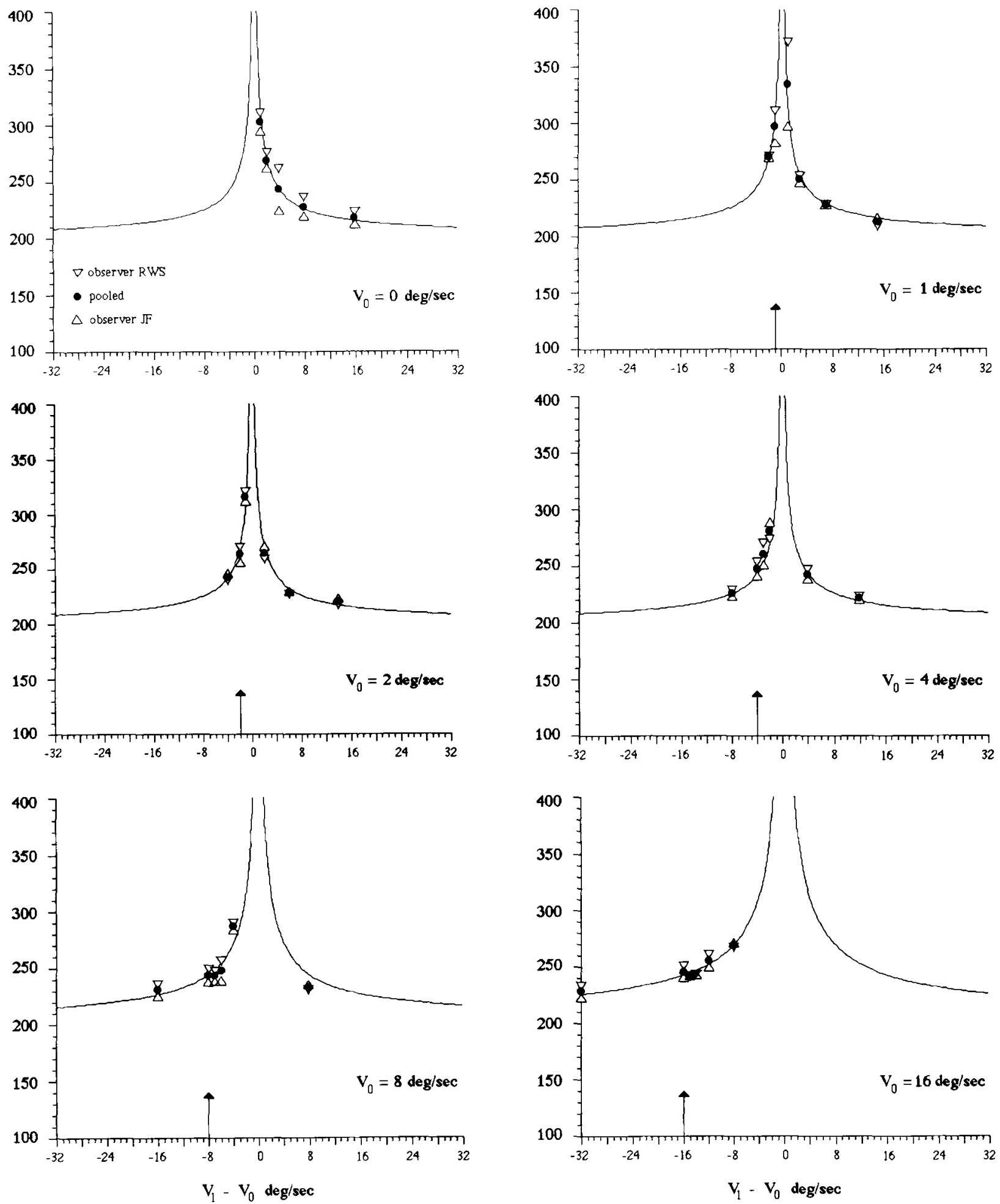

Figure 2. Mean RT as a function of $V_{1}-V_{0}$ for Observers J.F. and R.W.S. Six panels correspond to six different values of $V_{0}$. In the panels for $V_{0} \neq 0$, speed-increment data form the right branches of the curves; direction-reversal data are the leftmost points in each panel; motion-offset data are the points corresponding to the abscissa value of $-V_{0}$ (marked by arrows); and the rest of the left branches are formed by speed-decrement data. The left branch for the motion-onset data $\left(V_{0}=0\right)$ is shown for uniformity. The theoretical curves are presented separately in Figure 3. They are fitted to MRT values averaged over the 2 observers: mean quadratic approximation error is $6.9 \mathrm{msec}$; $S E M=3.4 \mathrm{msec}$. 


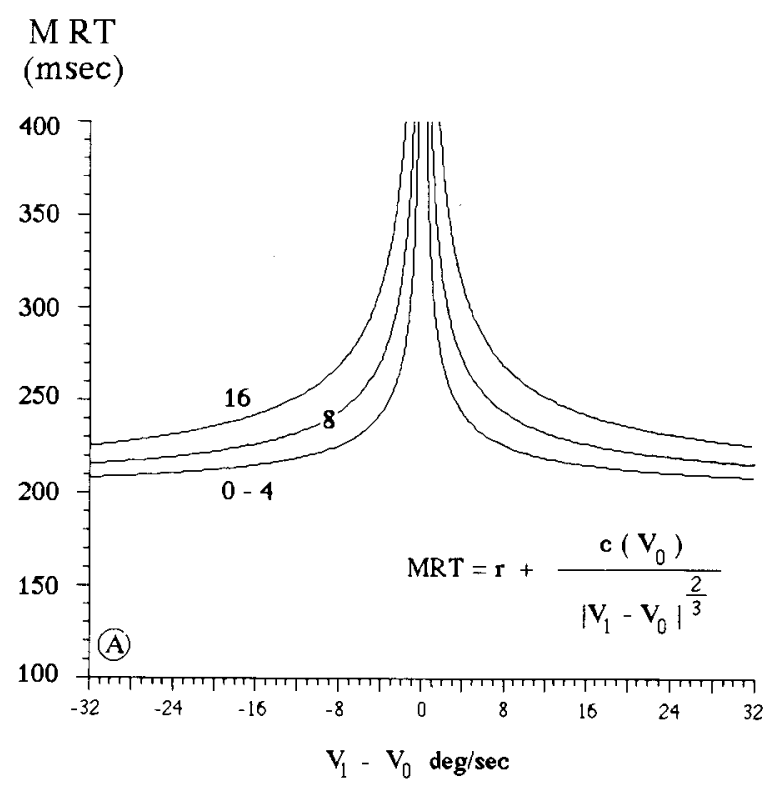

MEAN QUADRATIC

ERROR (msec)

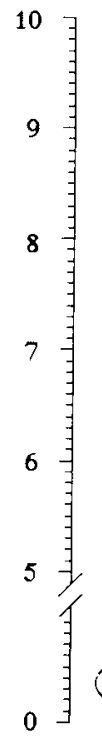

(B)
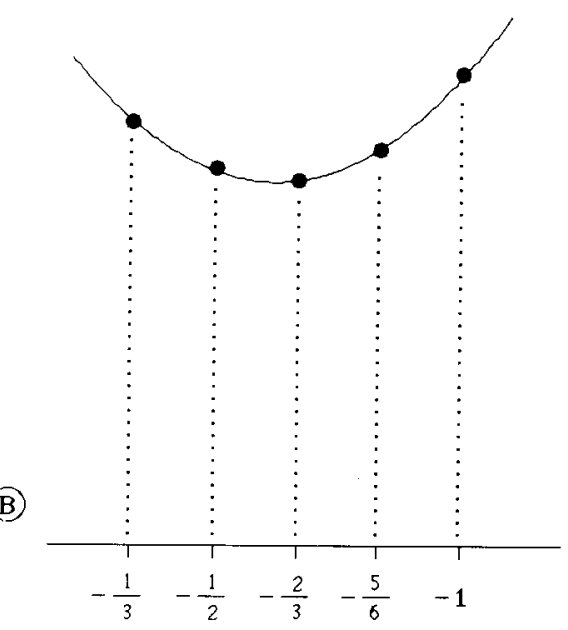

EXPONENT

Figure 3. (A) The theoretical curves of Figure 2: MRT $=r+$ $c\left(V_{0}\right)\left|V_{1}-V_{0}\right|^{-2 / 3}$, with $r=197 \mathrm{msec}, c(0)=c(1)=c(2)=c(4)=$ $0.115, c(8)=0.187, c(16)=0.287\left(\mathrm{sec}^{1 / 3} \mathrm{deg}^{2 / 3}\right)$. (B) The mean quadratic error of approximation for the MRTs of Figure 2 by $r+c\left(V_{0}\right)\left|V_{1}-V_{0}\right|^{-\beta}$ with different values of $\beta$, under the restriction $c(0)=c(1)=c(2)=c(4)$.

2. In ordering the various MRT values for a given $V_{0}$, only absolute values of velocity difference matter, $\mid V_{1}-$ $V_{0} \mid$, irrespective of whether they represent speed increments (right branches), speed decrements (left branches), direction reversals (the leftmost data points in each panel), motion onsets (the panel for $V_{0}=0$ ), or motion offsets (the points corresponding to the abscissa value of $-V_{0}$ in each panel). The Wilcoxon two-group test applied to the MRT pairs corresponding to equal decrements and increments from a common $V_{0}$ value (e.g., $[4,0]$ vs. $[4,8] \mathrm{dps}$ ) reveals no systematic difference between them (two-sided $p=.89, n=2$ observers $\times 4$ pairs $=8$ ). At the same time, the Goodman-Kruskal gamma for the association of MRT with $\left|V_{1}-V_{0}\right|$, computed separately for six different $V_{0}$ values and 2 observers, is -1 in 10 out of 12 cases (onesided $p=.001$ in $6 \times 5$ association, and for $V_{0}=0, p=$ .008 in $5 \times 5$ association). The two exceptions are $\mathrm{Ob}$ server R.W.S., $V_{0}=8(\gamma=-0.86, p=.008$, one inversion in $6 \times 5$ association) and $V_{0}=16(\gamma=-0.73$, $p=.014$, two inversions in $6 \times 5$ association).

3 . For a given value of $\left|V_{1}-V_{0}\right|$, MRT increases as $V_{0}$ increases from some value above $4 \mathrm{dps}$. No such trend is discernible for $V_{0}$ values between 0 and $4 \mathrm{dps}$; within this range, MRT is solely determined by $\left|V_{1}-V_{0}\right|$ (the Goodman-Kruskal gamma between MRT and $\left|V_{1}-V_{0}\right|$ computed over this range is -0.96 for J.F. and -0.94 for R.W.S., $p=.000,23 \times 11$ association).

The scatterplot in Figure 4 presents the results of the auxiliary experiment, in which we varied the number of dots in the display. MRT values corresponding to the same $\left(V_{0}, V_{1}\right)$ values but different numbers of dots $(200,100$, and 50) are plotted against each other pairwise. The vertical axes have been shifted with respect to each other for better readability. Any differences due to different numbers of dots are reflected in deviations of the data from the identity lines (the expected loci of data points if MRT

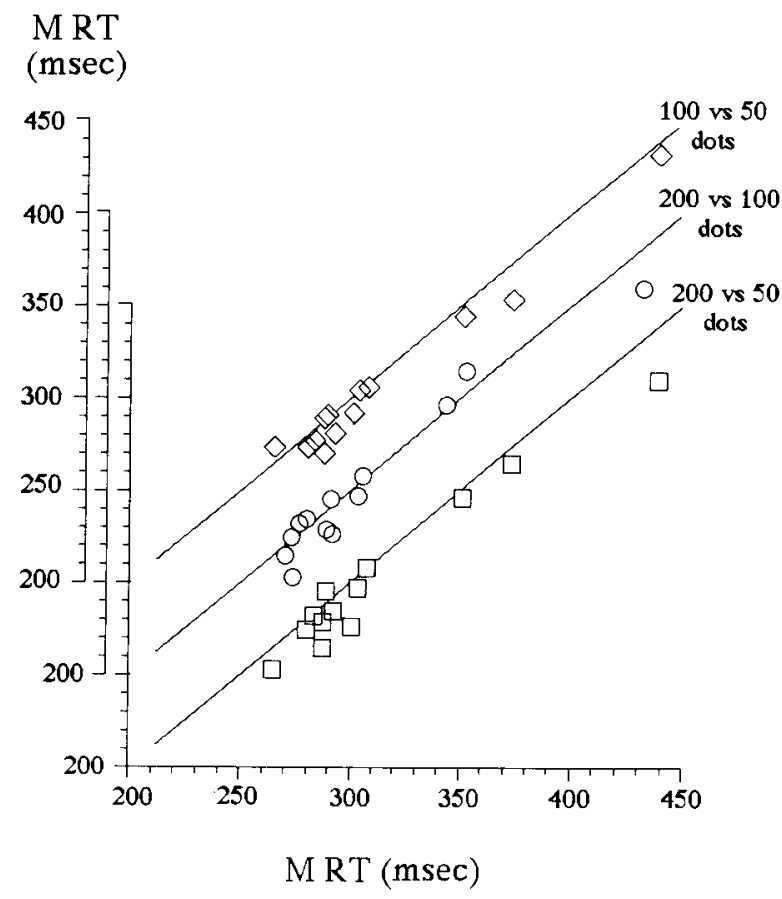

Figure 4. Mean RTs for 200-, 100-, and 50-dot displays plotted against each other. Each symbol represents MRTs corresponding to the same velocity pair but two different numbers of dots $(\mathrm{Ob}-$ server J.L.M.). The graphs are vertically shifted with respect to each other by 50-msec steps. The theoretical lines are unit slope zero intercept identity lines. 
did not differ at all with number of dots). Clearly there are no large systematic deviations, although the MRTs for 50 dots $\left(\mathrm{MRT}_{50}\right)$ do show a tendency to exceed the corresponding $\mathrm{MRT}_{100}$ and $\mathrm{MRT}_{200}$ (the Friedman rank sums are, respectively, $34.5,22.5$, and 21 in a $13 \times 3$ table, $p=$ $.015)$. This effect, however, is very small, as can be seen from the fact that lowering MRT $\mathrm{M}_{50}$ values by only 2 or $3 \mathrm{msec}$ would result in incrementing the Friedman test $p$ value to .125 and .232 , respectively. The mean quadratic deviation of the MRTs from the corresponding values of $\left(\mathrm{MRT}_{200}+\mathrm{MRT}_{100}+\mathrm{MRT}_{50}\right) / 3$ is $6.0 \mathrm{msec}$. We conclude that the large change from 50 to 200 dots has a remarkably small effect on MRT. Therefore, our principle results do not seem to be restricted to the particular number of moving dots used in the main experiment.

\section{Quantitative Analysis}

The ordinal-scale properties of the data discussed in the preceding section are simple consequences of the fact that the data can be well approximated by the theoretical curves shown in Figure 3A. For reasons that will be clear from the following text, the approximation was first sought for the motion-onset data $\left(V_{0}=0\right)$ in the form

$$
\operatorname{MRT}(0, V)=r+c|V|^{\beta},
$$

where $r$ is the mean value of $\mathbf{R}$ in Equation 1 (the velocityindependent component of RT), and $c$ is a positive constant that can be interpreted as the mean value of the detection criterion. This formula is compatible with the following special form of Equation 1:

$$
\mathbf{R T}(V)=f(\mathbf{C})|V|^{\beta}+\mathbf{R},
$$

where $f(\mathbf{C})$ is an arbitrary function. We found that virtually perfect approximation can be achieved when $\beta$ is set very close to $-2 / 3, r$ close to $0.2 \mathrm{sec}$, and $c$ close to 0.1 (note that given $\beta=-2 / 3, c$ is measured in $\sec ^{1 / 3} \mathrm{deg}^{2 / 3}$ ).

We found further that MRTs for $V_{0}=1-, 2-$, and 4-dps velocity changes fall on the same curve when plotted against $V=V_{1}-V_{0}$ :

$$
\operatorname{MRT}\left(V_{0}, V_{1}\right)=r+c\left|V_{1}-V_{0}\right|^{\beta},
$$

where $r, c$, and $\beta$ are as above. In this range of $V_{0}$, therefore, there appears to be a complete subtractive normalization (in the mean).

The MRT values for $V_{0}=8$ and $16 \mathrm{dps}$ fall above the curve just described, but they still can be reasonably well approximated by Equation 6 with the same values of $\beta(\approx$ $-2 / 3)$ and $r(\approx 0.2 \mathrm{sec}$ ) but higher values of $c$ (about 0.2 and $0.3 \mathrm{sec}^{1 / 3} \mathrm{deg}^{2 / 3}$ for 8 and $16 \mathrm{dps}$, respectively). Combining the results for all $V_{0}$ values used, we conclude that (in the mean) there is a strong subtractive normalization in detection of velocity changes, but that overall the normalization is not complete.

The curves shown in Figures 2 and $3 \mathrm{~A}$ were obtained as a result of a simultaneous approximation of all data points by

$$
\operatorname{MRT}\left(V_{0}, V_{1}\right)=r+c\left(V_{0}\right)\left|V_{1}-V_{0}\right|^{-2 / 3},
$$

under the restriction that $c\left(V_{0}\right)$ has the same value for $V_{0}$
$=0,1,2,4 \mathrm{dps}$. Figure 3B shows that under the same restrictions, the quality of approximation deteriorates if $\beta=-2 / 3$ in Equation 7 is changed in either direction. The absolute value of the deterioration, in the range of $\beta$ shown in Figure 3B, is rather small, however. Figure 3B, therefore, only illustrates the optimality of choosing $\beta=$ $-2 / 3$, but does not allow one to definitively reject other values between -1 and $-1 / 3$. Equation 7 suggests (though does not prove) the following special form of Equation 3:

$$
\mathbf{R T}\left(V_{0}, V_{1}\right)=f(\mathbf{C})\left|V_{1}-V_{0}\right|^{\beta}+\mathbf{R},
$$

where $\beta \approx-2 / 3, f(C)$ generally depends on $V_{0}$, and $\mathbf{R}$ is a $V_{0}$-independent random variable.

An important characteristic of the quality of approximation provided by Equation 7 is that a variety of more general models, of which Equation 7 is a special case, yield only negligible gain in fit. For example, models in which both $c$ and $r$ are allowed to depend on $V_{0}$, or in which $\left|V_{1}-V_{0}\right|^{\beta}$ is replaced with $\left.\left.\left|V_{1}^{\alpha}-\operatorname{sgn}\left(V_{0}\right)\right| V_{0}\right|^{\alpha}\right|^{\beta}$, and other reasonable generalizations we tried, reduced the quadratic approximation error by less than $0.5 \mathrm{msec}$. The values of the parameters of these more general models either converge to those of Equation 7 (e.g., $\alpha$ is set close to 1 at the optimum), or show an obviously chaotic dependence on $V_{0}{ }^{1}$

Figure 5 demonstrates, in a format different from that of Figure 2, the applicability of Equation 7 to the results of our auxiliary experiment (see the preceding section and Figure 4). One can see that all MRTs, irrespective of what kind of change is being detected, after appropriate normal-

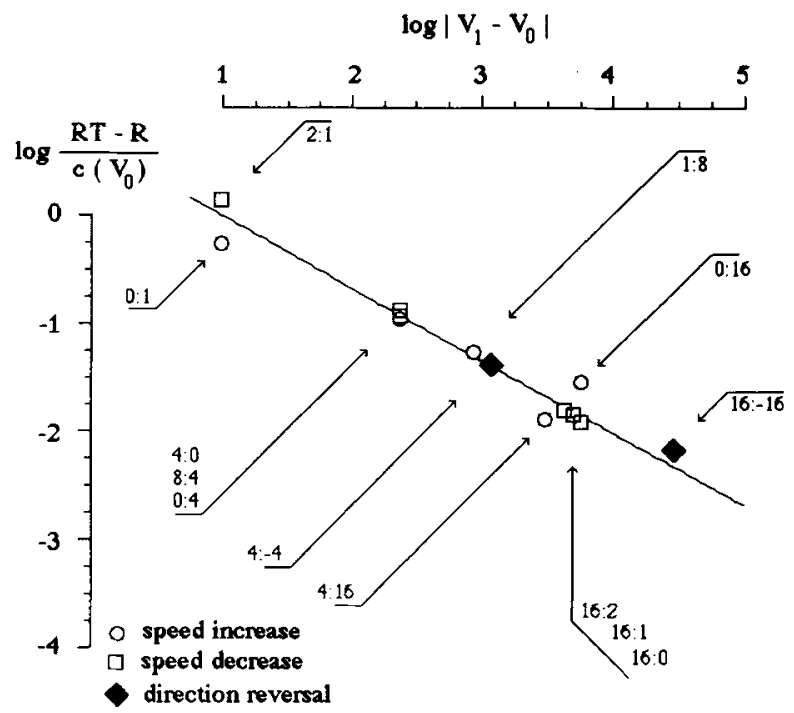

Figure 5. MRT versus $\left|V_{1}-V_{0}\right|$ in $\log -\log$ plots $\left(\log _{e}\right)$ for Observer J.L.M., auxiliary experiment (the MRTs are averaged across 200-, 100-, and 50-dot displays; see Figure 4). Different symbols correspond to speed increments (including motion onsets), speed decrements (including motion offsets), and direction reversals. The velocity pairs are identified numerically. The theoretical straight line is $\log \left[(\mathrm{MRT}-r) / c\left(V_{0}\right)\right]=-(2 / 3) \log \left|V_{1}-V_{0}\right|$, where $r=238 \mathrm{msec}$, $c(0)=c(1)=c(2)=c(4)=0.165, c(8)=0.260, c(16)=0.325$ $\left(\sec ^{1 / 3} \mathrm{deg}^{2 / 3}\right)$. Mean quadratic approximation error is $0.146 \log _{e}$ units. 
ization (subject to the same restrictions as above), fall on a straight line with a slope of $-2 / 3$ when plotted against $\left|V_{1}-V_{0}\right|$ in $\log$-log coordinates.

\section{DISCUSSION}

\section{Mechanisms of Motion-Onset Detection}

Our experimental data indicate a strong subtractive normalization of visual motion in detection of velocity changes; the visual system seems to effectively reduce this task to that of motion-onset detection. Obviously, a theoretical interpretation of this finding critically depends on one's understanding of possible mechanisms of motion-onset detection. This is the subject of this section, in which we attempt to justify the negative-exponent power function relating MRT to $V$ (Equation 5), account for the exponent's empirical value ( $\beta \approx-2 / 3$ ), and provide a theoretical interpretation for parameter $c$. In accordance with our distinction between general-purpose encoding computations and special-purpose detection computations, we begin with a brief and admittedly simplistic discussion of the hypothetical encoding network.

Since Reichardt's (1961) work on the insect's optomotor system, most psychophysical models of visual motion encoding have been based on the idea of quadruple computations: the response of an elementary motion encoder is based on characteristics of four luminance profiles sampled from two spatial areas at two moments of time (see Nakayama, 1985, for a review; see also van Doorn \& Koenderink, 1983; van Doorn, Koenderink, \& van de Grind, 1984; van Santen \& Sperling, 1984, 1985). Denoting the centers of the two areas (along the horizontal axis) by $X$ and $X+\Delta X$, the sampling moments by $T$ and $T+\Delta T$, and assuming that the two areas are identical except for the shift, a quadruple computation is formally an operation on a $2 \times 2$ matrix formed by the luminance profiles corresponding to $(X, T),(X+\Delta X, T),(X, T+\Delta T)$, and $(X+\Delta X$, $T+\Delta T)$. The parameters $\Delta X$ and $\Delta T$ are referred to as, respectively, the spatial span and temporal span of an encoder. Without getting into detail, when applied to a rigid motion of a sharply localized pattern, many formally different quadruple operations (including the determinanttype computation proposed by Reichardt) are equivalent to the following simple rule. An encoder with spatiotemporal span $(\Delta X, \Delta T)$ can only be activated if

$$
x(t+\Delta T)-x(t)=\Delta X,
$$

where $x(t)$ is as defined above: spatial position $x$ at moment $t$ of a fixed point taken on the moving target. Assume, as an idealization, that if an encoder with parameters $(\Delta X, \Delta T)$ exists, then there are sufficiently many such encoders, so that (1) the centers $X$ of their left (or right) spatial areas cover the visual space densely, and (2) the luminance profiles within any such area can be sampled at any moment $T$. Then the pattern shift described in Equation 8 is both sufficient and necessary for the activation of a $(\Delta X, \Delta T)$ encoder. ${ }^{2}$
We now consider three simple schemes of motiondetection computations that can be performed on the outputs of the network of motion encoders. We choose these three schemes not as a result of a systematic or exhaustive analysis of possibilities, but simply because they lead to motion-detection models that have been proposed in the literature. The descriptions of each of the models contain further assumptions concerning the network of motion encoders.

A Single-Encoder Scheme 1 (the critical-distance model). The simplest assumption one can make is that motion detection occurs if and only if at least one motion encoder is activated. This detection rule implies the following. Consider a time-position function $x(t)$. At any moment $t$, one can compute from this function the set of the spatiotemporal distances $(\Delta X, \Delta T)$ for all pairs of spatiotemporal locations the pattern has passed through before the moment $t$. For example, if a pattern rests for an indefinitely long period, and at a zero moment begins moving with a constant velocity $V$ (the situation we had in our motion-onset-detection trials), then $t$ units of time later the set of the pairwise spatiotemporal distances will contain all $(\Delta X, \Delta T)$ constrained by $\Delta X \leq V t$. Another example that will be useful for our discussion is detection of instantaneous displacements. If a pattern rests for an indefinitely long period, and at a zero moment shifts instantaneously to another position (say, $A$ spatial units to the right), where it stays, then $t$ units of time later the set of the pairwise spatiotemporal distances will consist of all $(\Delta X, \Delta T)=(A, \Delta T)$. Now, if an observer fails to detect such a motion before some moment $t$, then this means that the network does not contain motion encoders whose $(\Delta X$, $\Delta T$ ) parameters match at least one of the pairwise spatiotemporal distances in the set just described.

Suppose, for example, that the network only contains encoders with $\Delta X \geq \Delta X_{\min }$ (a minimum spatial span, with no restrictions on temporal span $\Delta T)$. Then $x(t)$ will be detected precisely at the moment $t$ when for the first time $x(t)-x(t-\Delta T)=\Delta X_{\min }$ for some value of $\Delta T$. Thus, in the case of a uniform motion starting at a moment zero, the detection will occur as soon as $V t=\Delta X_{\min }$. One can recognize here a prediction of the critical-distance model, mentioned earlier. The model provides a rather poor account of known threshold and RT data (including those reported in this paper). It is known, for example, that the minimum excursion required to make a horizontal motion just visible depends critically on other kinematic parameters, such as translation time for unidirectional motions (Johnson \& Scobey, 1980; Scobey \& Johnson, 1981) and waveform and frequency for oscillations (Dzhafarov \& Allik, 1984; Dzhafarov, Allik, Linde, \& Piastolov, 1981; Nakayama \& Tyler, 1978; Tyler \& Torres, 1972). When applied to detection of instantaneous displacements, the model predicts that no detection will occur if the displacement magnitude $A$ is less than $\Delta X_{\min }$, but that detection will be instantaneous once $A$ exceeds $\Delta X_{\min }$. Attempts to modify the model in order to exclude this obviously wrong 
prediction (MRT is in fact a linear function of $A^{-2}$, as discussed below) would probably lead to a version of the model we consider next.

A Single-Encoder Scheme 2. This model was proposed by van den Berg and van de Grind (1989) on the basis of extensive research done by van Doorn, Koenderink, and van de Grind (van de Grind, Koenderink, \& van Doorn, 1983, 1986; van Doorn \& Koenderink, 1982a, 1982b, $1983,1984)$. According to the model, the spatial and temporal spans $\Delta X$ and $\Delta T$, in the network of motion encoders, are not paired in an all-to-all fashion. Instead, for any value of the spatial-to-temporal span ratio, $v=\Delta X / \Delta T$, there exists only one pair $(\Delta X, \Delta T)$ with this ratio. This encoder is termed a detector of velocity $v$. Results presented in van Doorn \& Koenderink (1982a, 1982b, 1983, 1984) show that the relation between $(\Delta X, \Delta T)$ and their ratio $v$ can be described by two power functions,

$$
\Delta X=a v^{\beta+1} \text { and } \Delta T=a v^{\beta},
$$

where $a$ is a positive constant and $\beta$ is estimated between -0.4 and -0.5 . Equation 9 is, of course, a parametric equivalent of the explicit formula

$$
\Delta T=c \Delta X^{\alpha},
$$

where $c$ is a positive constant and $\alpha=\beta /(\beta+1)$; hence, its value should be estimated between $-2 / 3$ and -1 . For applications involving detection of motion onset (a constant velocity $V$ following a period of rest), it is simpler to use Equation 9.

The predictions concerning MRT are derived in the following way. (1) Assume that a motion encoder is activated only if a temporal change occurs at or close to its first sampling moment (in van den Berg \& van de Grind, 1989 , this important assumption is implicit); (2) then the first sampling moment of the encoder tuned to $V$ will coincide with the moment of motion onset; (3) the second sampling will occur $\Delta T=a V^{\beta}$ units of time later and $\Delta X=$ $a V^{\beta+1}$ spatial units apart; (4) the two samples will match and the encoder tuned to $V$ will be activated; (5) no encoder with $\Delta T<a V^{\beta}$ will achieve a match, and hence, the encoder tuned to $V$ will be the first to respond (in fact, the only one to respond). As a result, the $V$-dependent RT component will equal $\Delta T=a V^{\beta}$, with the exponent between -0.4 and -0.5 .

This prediction provides a good account of the motiononset data presented in this paper (Figure 1, panel $V_{0}=0$ ). Even though the optimal exponent appears closer to $-2 / 3$ than to -0.5 (see Figure $3 \mathrm{~B}$ ), the difference is too small to warrant a preference judgment. Figure 6 presents some additional data sets on MRT to motion onset available from three earlier published papers and one unpublished experiment. In all these cases, the optimal exponent is close to $-2 / 3$ (surprisingly, even for Collewijn's, 1972, data on optokinetic nystagmus in the rabbit). The stability of this data pattern does exclude models of the critical-distance type (see Troscianko \& Fahle, 1988, for a contrary opinion). The model of van den Berg and van de Grind (1989), however, cannot be rejected on the basis of these motion- onset data, because the exponent of -0.5 provides an almost equally good approximation (moreover, the value -0.4 or -0.5 for $\beta$ in Equation 9 is an empirical estimate derived from independent experiments, and one cannot exclude the possibility that more precise measurements could bring this value even closer to $-2 / 3$ ).

A more serious problem is encountered when the model is applied to detection of instantaneous shifts. Figure 7 presents MRT values plotted against the magnitude of the shift, which is almost perfectly approximated by a power function with the exponent of -2 . This value has been verified by a thorough analysis of RT versus magnitude of shift, presented in Dzhafarov (1992), and it holds not only for the means but also for all percentiles of RT distributions that are not too close to the distribution margins. The approximation of the data in Figure $7 \mathrm{~A}$ by a power function with the exponent of -1 or less provides a fit that is inferior to the one shown in the figure by a factor of 10 or more (in terms of time-dimensioned mean quadratic errors, see Figure 7B). The predictions of the model proposed by van den Berg and van de Grind (1989) can easily be derived from Equation $9 *$ using the same logic as above. The predicted exponent equals $\alpha$ in Equation 9*; that is, it falls between $-2 / 3$ and -1 , which is clearly unacceptable. There seems to be no way to improve this prediction without simultaneously worsening the prediction quality for uniform motion onset.

The model also has difficulties in accounting for the known data on kinematic thresholds. For example, minimum amplitude of oscillation required for just visibility of a horizontal motion depends on the oscillation waveform (Dzhafarov \& Allik, 1984; Dzhafarov et al., 1981; Nakayama \& Tyler, 1978; Tyler \& Torres, 1972), contrary to what the model predicts. A modification of the model that might bring it closer to such data would require shifting the focus of the analysis from the relationship between spatial and temporal spans to temporal changes within the sampling areas; sampling in such a modification would have to be treated as an extended computation. A more detailed discussion of these issues is beyond the scope of this paper. The reason for mentioning the difficulties encountered by the model in question is not to dismiss it, but rather to justify considering the alternative approach to which we turn next.

A mass activation scheme. The approach is to consider motion detection as a computation over the distributed pattern of activations within the network of motion encoders, rather than as a result of activation of an encoder (or a group of encoders) with a given spatiotemporal span. The assumptions are that to a first approximation, all sampling areas (defined in external rather than retinal coordinates) are essentially identical, that they cover the visual field densely, and that sampling within any given area occurs within negligibly short intervals rapidly following each other. The sampling areas are spatially interconnected in an all-to-all fashion, or at least within a range of intercenter distances from zero to some large value. Moreover, each spatial connection contains a set of parallel lines with built- 

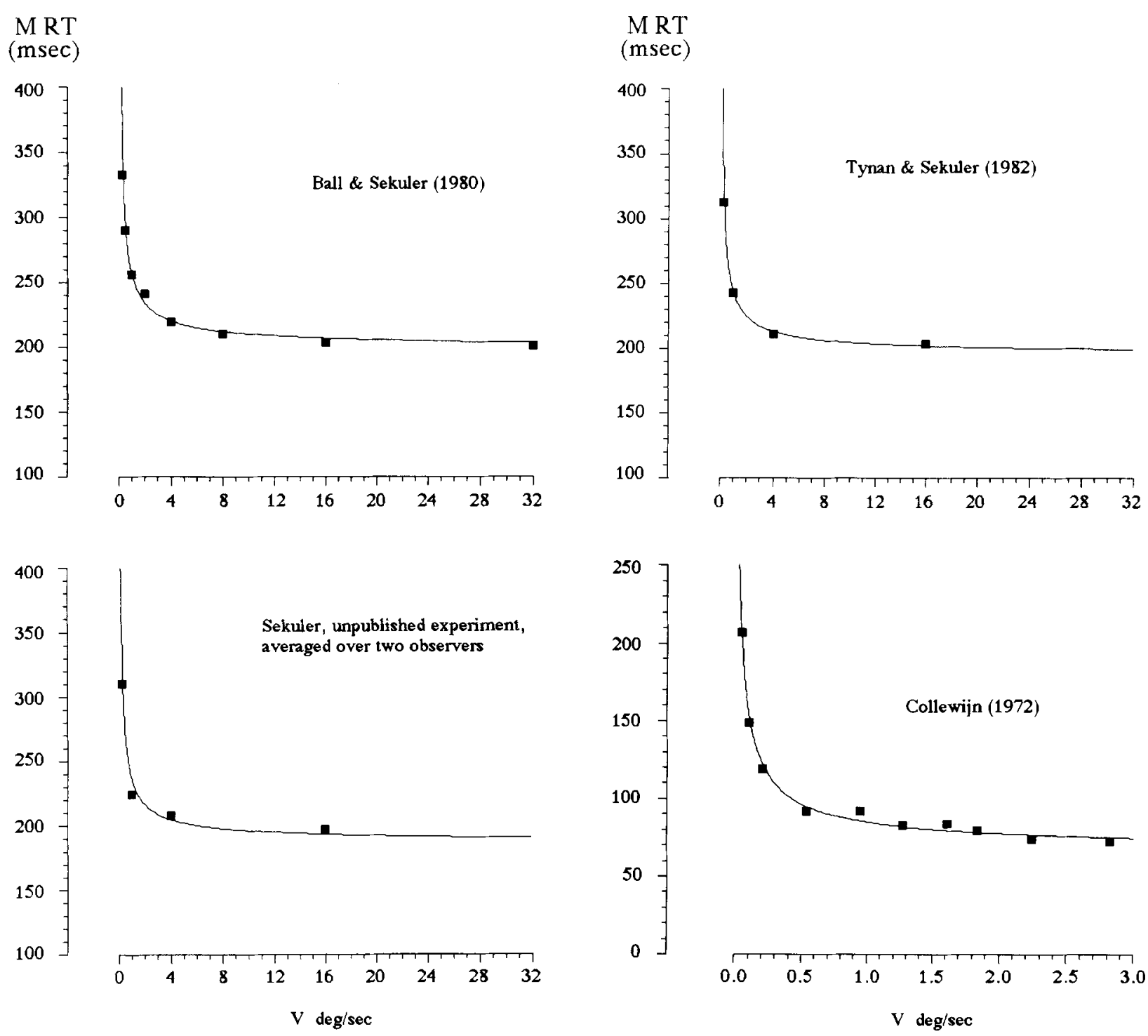

Figure 6. MRT to motion onset as a function of motion speed $V$, taken from previous studies. The bottom right panel represents the optokinetic nystagmus latency in the rabbit. All data are approximated by negative-exponent power functions with the exponent of $-2 / 3$.

in delays ranging from zero to some large value. Again, to a first approximation, the delay values densely cover the interval just mentioned (no nonnegligible gaps between successive delay values), and all delay values are associated with all spatial connections. In this network, every pair of spatial areas (say, centered at $x$ and $x+\Delta X$ ) and every pair of sampling moments $(t$ and $t+\Delta T)$ define one elementary quadruple computation. The result of this computation will be activation (say, Boolean 1) if and only if Equation 8 is satisfied; otherwise, the result will be no activation (Boolean 0$)$. Any position-time function $x(t)$, therefore, at a moment $t$ will be encoded by all $(\Delta X, \Delta T)$ such that $x(t)-x(t-\Delta T)=\Delta X$.

The elementary quadruple computations can be conceptually grouped in many different ways: by their spatial span
$\Delta X$ ("spatial shift detectors"), by their temporal span $\Delta T$ ("time delay detectors"), by their spatial-to-temporal span ratio $v=\Delta X / \Delta T$ ("velocity detectors"), or by any other function of $(\Delta T, \Delta X)$. There seems to be no universal, context-independent reason for preferring one possible conceptual grouping to another.

Under the assumptions made, there always will be a mass activation within the network at any moment for any motion, so a reasonable motion-detection computation should be based on some form of temporal summation (or averaging) across the activated units. In such averaging, each quadruple computation can be taken with some weight $w$, which generally may depend on its defining spatiotemporal parameters: $w=w(t, x, t+\Delta T, x+\Delta X)$, that is, the two sampling moments and two areas of the computation. As- 


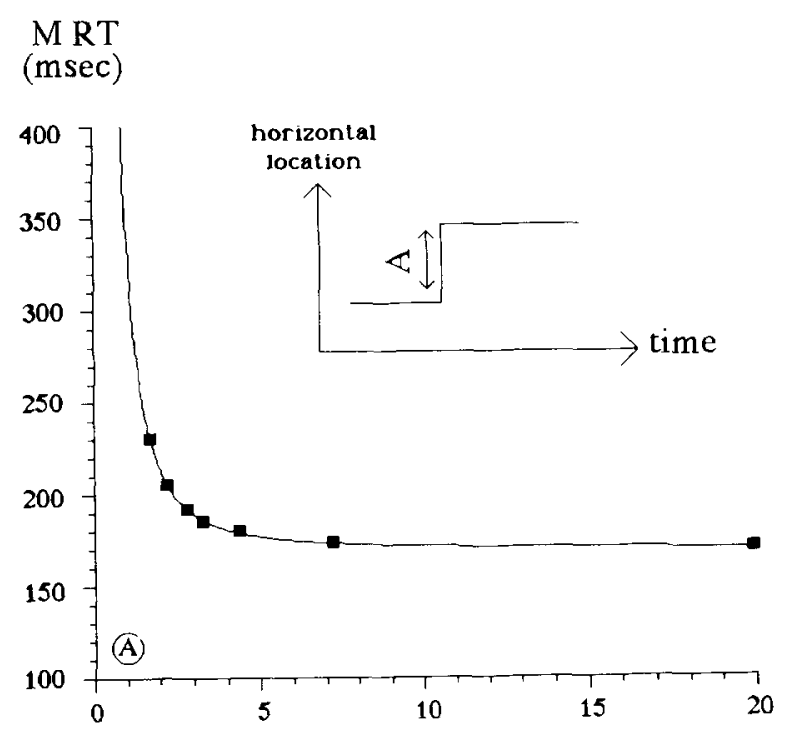

A, min arc

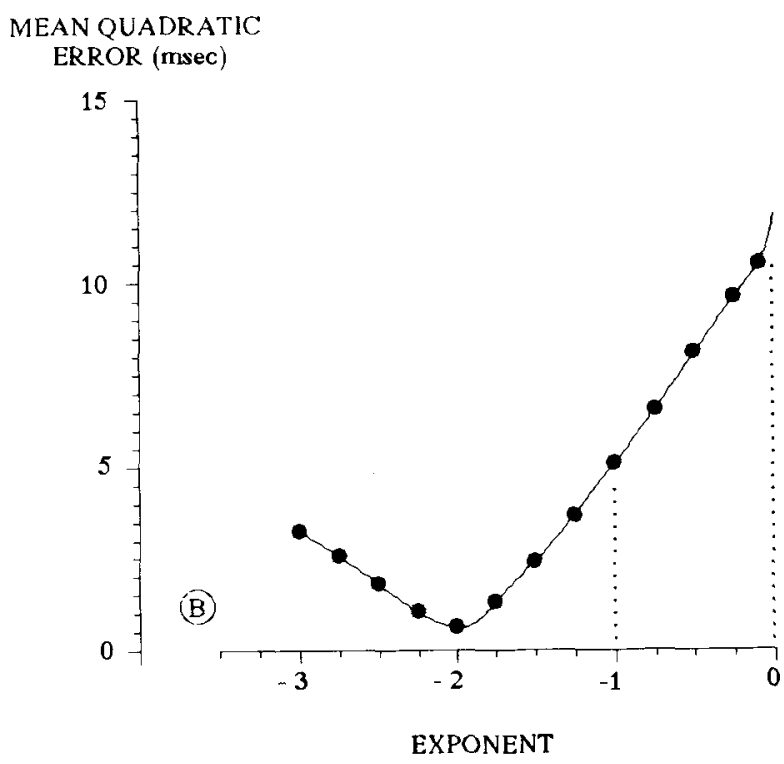

Figure 7. MRT to instantaneous displacements versus displacement magnitude, from Dzhafarov (1992). The theoretical curve in panel $A$ is a power function with the exponent of $-2, r+\mathrm{cA}^{-2}$. Panel $B$ shows the mean quadratic error of approximation for the same MRTs by $\boldsymbol{r}+\mathbf{c A}^{-\beta}$ with different values of $\beta$.

suming spatiotemporal homogeneity, the absolute coordinates can be dropped and the weight becomes a function of the spatiotemporal span only:

$$
w=w(\Delta X, \Delta T) \text {. }
$$

The result of averaging at some moment $t$ then can be approximated by the following expression:

$$
\begin{aligned}
& E(t)= \\
& \tau^{-2} \int_{t-\tau}^{t} \int_{t-\tau}^{t} w\left(t_{2}-t_{1}, x_{2}-x_{1}\right) B\left(t_{2}-t_{1}, x_{2}-x_{1}\right) d t_{2} d t_{1},
\end{aligned}
$$

where $\tau$ is the effective duration of the summation window, and $B$ is the Boolean value of the activation (ensuring that only activated encoders are being counted). Motion is detected when this value reaches some criterion value $\mathbf{C}$, a random variable.

Dzhafarov and Allik (1984; Dzhafarov, Allik, \& Linde, 1983) proposed a particularly simple form for the weighting function $w$ :

$$
w(\Delta X, \Delta T)=(\Delta X)^{2} .
$$

The general idea is that in the set of activated encoders, those with larger spatial spans convey more evidence for the presence of motion than those with smaller spatial spans. It is also natural to assume that encoders with small spatial spans are to a greater extent false-alarm prone, for example, due to small-amplitude involuntary eye movements that have not been compensated for. Averaging in general is an effective means for suppressing spurious activations, but its effectiveness is enhanced by assigning greater weights to larger spatial spans. The temporal spans of motion encoders, according to Equation 12, are not relevant provided they are within the summation window $(t, t-\tau)$.

The particular form of the monotonic dependence of $w$ on $\Delta X$ chosen, $w(\Delta X)=(\Delta X)^{2}$, directly follows from the analysis of RT to instantaneous displacements (Dzhafarov, 1992; see Figure 7). As we show in the Appendix, MRT in this case is approximately a linear function of $w(A)^{-1}$, the reciprocal of the weighting function for a spatial span equal to the displacement magnitude. MRT is empirically shown to be a linear function of $A^{-2}$, so the function $w(A)$ has to be $A^{2}$. This result has a certain mathematical appeal, because in the case of squaring, Equation 11 is simply the moving variance of the spatial positions passed through within a temporal window of length $\tau$ :

$$
E(t)=\sigma^{2}\{x(u): t-\tau<u<t\} .
$$

The function $E(t)$ therefore can be appropriately termed kinematic power. A numerical illustration of how the kinematic power is computed from the activation pattern of the postulated network of motion encoders in given in Figure 8.

As mentioned above, spatial positions of the sampling areas are assumed to be defined in external rather than retinal coordinates. This implies the existence of some form of compensation for eye movements prior to the motionencoding network. Less than perfect compensation will result in localization errors that will modify the kinematic power values. It is shown in the Appendix, however, that under certain assumptions, localization errors do not change the formal structure of the model, only $\mathbf{C}$ now has to be interpreted as the difference between a random criterion and the localization error variance (perhaps normalized by another noise term; see Appendix for details). For motion detection to occur, the kinematic power computed in external error-free spatial coordinates (Equation 13) has to reach this difference value.

The model successfully accounts for kinematic threshold data relating the minimum excursion required for just 


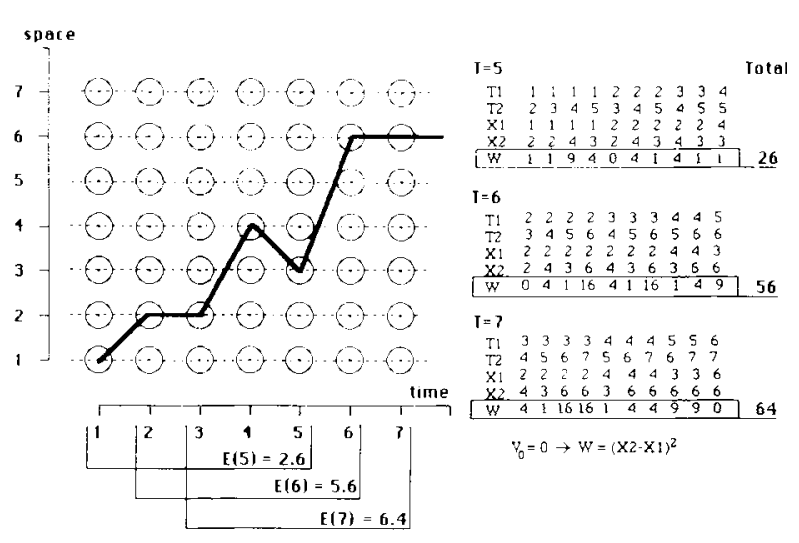

Figure 8. A discretized version of kinematic power computations in motion-onset detection. Circles represent seven evenly spaced subfields at seven evenly spaced sampling moments. Any four circles forming a rectangle (i.e., defined by two sampling moments, $T 1$ and $T 2$, and two area centers, $X 1$ and $X 2$ ) represent one elementary quadruple computation yielding Boolean 1 (activation), if and only if the time-position function (shown by the thick line) passes through its lower left and upper right corners. Horizontal dashed lines show the reference time-position function (in this case, no motion) from which the ongoing motion is to be distinguished. In this example, we assume that the summation window $(\tau)$ is five sampling moments long. The tables to the right of the diagram list all activated quadruples $(T 1, T 2, X 1, X 2)$ whose sampling moments ( $T 1$ and $T 2)$ fall between some moment $T(=5,6$, or 7$)$ and moment $T-\tau=$ $T-5+1$. The bottom row shows the weights with which the activations are being summated, $W=(X 2-X 1)^{2}$. Kinematic power $E$ at moment $T$ is the mean weight between $T$ and $T-5+1$, that is, the weight total divided by the number of activated quadruples (10 in our case).

visibility of motion to the waveform, frequency, or duration of the motion (Dzhafarov \& Allik, 1984; Dzhafarov et al., 1981). ${ }^{3}$ When applied to the detection of onset of uniform motion (constant velocity $V$ following a period of rest), the model predicts that MRT is a negativeexponent power function of $V$, with the exponent value of $-2 / 3$ (Equation 5; Figure 2, panel $V_{0}=0$ ). A formal derivation of this fact is given in the Appendix, where it is also shown that $c$ in Equation 5 represents the mean value of a random variable monotonically related to the criterion $\mathbf{C}$.

\section{Readjustment of Weights \\ Mechanisms of strong subtractive normalization.} One appealing consequence of treating motion-onset detection as a special-purpose computation (specifically, weighted averaging or summation over the pattern of activated elementary motion encoders) is that other motionrelated tasks can, in principle, be solved by simply readjusting the weights or summation intervals while using the same algorithm of summation (averaging). In particular, it is easy to see how the strong subtractive normalization indicated by our experiments can be computationally realized in this way. The weighting function following the $V_{0}$ phase should be readjusted to become (compare with Equation 12)

$$
\begin{aligned}
w_{\bmod }\left(\Delta X, \Delta T, V_{0}\right) & =w\left(\Delta X-\tilde{\mathbf{V}}_{0} \Delta T, \Delta T\right) \\
& =\left(\Delta X-\tilde{\mathbf{V}}_{0} \Delta T\right)^{2},
\end{aligned}
$$

where $\tilde{\mathbf{V}}_{0}$ is an "estimate" of $V_{0}$ computed within some period during the $V_{0}$ phase of motion. Based on A. B. Sekuler et al. (1990), this period could be about $0.5 \mathrm{sec}$ (see our introductory discussion).

Provided that the summation (averaging) algorithm otherwise remains the same (Equation 11), the moving variance of Equation 13 is transformed into

$$
E\left(t, V_{0}\right)=\sigma^{2}\left\{x(u)-\tilde{\mathbf{V}}_{0} u: t-\tau<u<t\right\} .
$$

Applying this computation to the two-velocity motion, $V_{0} t \rightarrow V_{1} t$, and assuming that $\tilde{\mathbf{V}}_{0} \approx V_{0}$, one gets Equation 7 and hence accounts for the strong subtractive normalization. A numerical illustration of how the modified kinematic power is computed to detect a change in a unitvelocity motion is given in Figure 9.

One can think of several possible computational schemes for $\tilde{\mathbf{V}}_{0}$ that would be compatible with our model-we mention two. A straightforward possibility follows from the fact that by the end of the summation time $\tau$, the kinematic power $E(t)$, computed according to Equation 13 within the $V_{0}$ phase, stabilizes at the level proportional to $V_{0}^{2}$ (see Equation A2 in the Appendix). As a result, a normalized square root of this value can be taken as $\tilde{\mathbf{V}}_{0}$. (Note that the summation algorithm in our model, Equation 13, could be formulated directly in terms of the moving standard deviation of spatial positions, $E(t)^{0.5}$, rather than moving variance. Note also that the value of the summation time, $\tau$, has been estimated from kinematic threshold data to be on the order of $0.5 \mathrm{sec}$; Dzhafarov \& Allik, 1984; Dzhafarov, Allik, \& Linde, 1983.) Another possibility is that $\tilde{\mathbf{V}}_{0}$ is computed as an average of the spatial-to-temporal
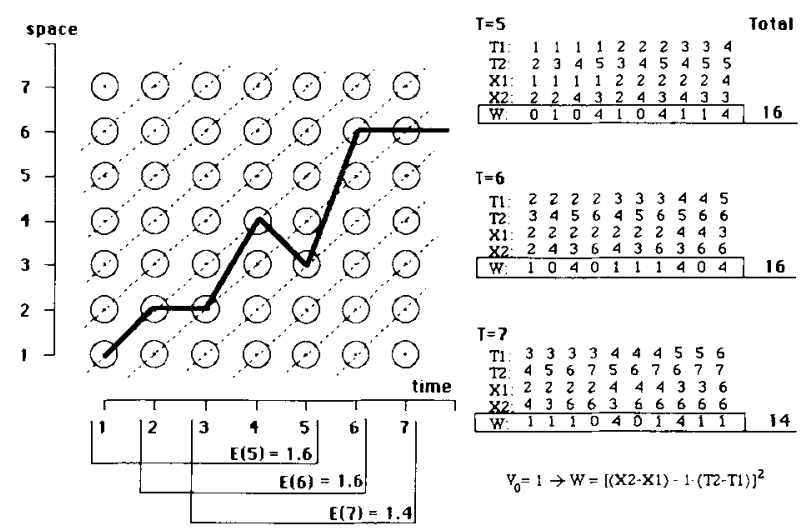

Figure 9. A discretized version of kinematic power computations in velocity-change detection. The network, the time-position function, and the summation window are the same as in Figure 8 . The ongoing motion is to be distinguished from a unit-velocity motion, $V_{0}=1$ (time-position function shown by dashed lines). The activated quadruples are, of course, the same as in Figure 8, but the weights here are computed by Equation 14: $\left[(X 2-X 1)-\tilde{V}_{0}(T 2-T 1)\right]^{2}$; in this example we assume that $\tilde{V}_{0}=V_{0}=1$ precisely. 
span ratios, $\Delta X_{0} / \Delta T_{0}$, of the encoders activated during the $V_{0}$ phase. The average could be computed, for example, as the mean spatial span of the activated encoders weighted by their temporal spans.

In our analysis of the experimental data (and in Figure 9), we have assumed that $\tilde{\mathbf{V}}_{0}$ equals $V_{0}$ precisely. More realistically, however, $\tilde{\mathbf{V}}_{0}$ is subject to random fluctuations, and it could even be slightly biased with respect to the true value of $V_{0}$ (a large bias would result in a high value of the modified kinematic power at the end of the $V_{0}$ phase, leading to a high rate of very short and premature false alarms, which we did not observe in our experiments). Admitting even moderate variability in $\tilde{\mathbf{V}}_{\mathbf{0}}$ would considerably increase the "fitability" of our model compared with that demonstrated in Figures 2 and 5, thereby lowering the model's empirical falsifiability. (The fitting procedure itself would also change dramatically - an observation that can be added to the comments made in Note 2.) It is fortunate, therefore, that the model provides a reasonable first-order approximation without such considerations of variability being utilized. These considerations, however, might be necessary in order to account for the empirical fact that the parameter $c$ in Equation 7 increases at higher values of $V_{0}$.

It is shown in the Appendix that the parameter $c$ is the mean value of a random variable $(3 \mathbf{C} \tau)^{1 / 3}$, a monotonic function of both criterion (corrected for noise factors) and summation time (the latter could also be considered a random variable, but for simplicity we have not done so). It is clear then that the observed increase in $c\left(V_{0}\right)$ that occurs at velocities of 8 and $16 \mathrm{dps}$ can be equally attributed to an increase in the mean criterion level and an extension of the summation time. There is no way to decide between these two possibilities on the basis of MRT values only. Both manipulations can counteract the effect of computational errors in weight readjustments and help to keep discriminability of signal from noise constant.

A question arises: If the value of $c\left(V_{0}\right)$ is determined primarily by the level of variability (computational error) in setting the value of $\tilde{\mathbf{V}}_{0}$, then why does this level increase at high velocities while remaining roughly constant at lower velocities? We will briefly discuss one possible solution.

Consider a single dot that appears at one border of a screen, moves toward another border, and disappears when it reaches it. In the two computational schemes mentioned, the adjustment of $\tilde{\mathbf{V}}_{\mathbf{0}}$ requires some time, which we denote by $\theta$. Again, for simplicity we do not consider $\theta$ to be a random variable. In the first scheme, based on kinematic power, $\theta$ cannot be less than $\tau$, the summation time for $E(t)$. In the second scheme, based on spatial-to-temporal span ratios, $\theta$ cannot be less than the maximal temporal span in the subpopulation of motion encoders utilized in the computation. As a result, if a velocity change, $V_{0} t \rightarrow V_{1} t$, occurs while the moving dot is within the distance of $V_{0} \theta$ from the border at which it first appeared, then the value of $\tilde{\mathbf{V}}_{0}$ cannot be set equal (or close) to $V_{0}$, as intended; indeed, the adjustment of $\tilde{\mathbf{V}}_{\mathbf{0}}$ will now include a portion of time after motion velocity has already changed to $V_{1}$. For example, in the spatial-to-temporal span ratio scheme, the averaging will include encoders with $\Delta X_{0} / \Delta T_{0}$ equal (or close) to $V_{0}$, but also those with $\Delta X_{0} / \Delta T_{0}$ equal (or close) to $V_{1}$; the same will be true for the kinematic power scheme. Let us term such adjustments of $\tilde{\mathbf{V}}_{0}$ "defective," as opposed to "normal" adjustments-those that take place entirely within the $V_{0}$ phase of motion, that is, when a velocity change occurs after the dot has moved beyond the distance $V_{0} \theta$.

In a multiple-dot constant-flow display, as in our experiments, some proportion of dots will be within the $V_{0} \theta$ distance from their starting points at any moment of time, irrespective of how long the movement has lasted. Figure 10 illustrates and quantifies this fact for circular displays. The vertical axis of the graph and the shaded areas in the diagrams could be interpreted as the proportion of "defective" adjustments of $\tilde{\mathbf{V}}_{0}$, if these adjustments were made separately for different dots. Throughout this paper we have carefully avoided, due to the lack of relevant empirical information, a discussion of possible cooperative algorithms pooling the results of the postulated computa-

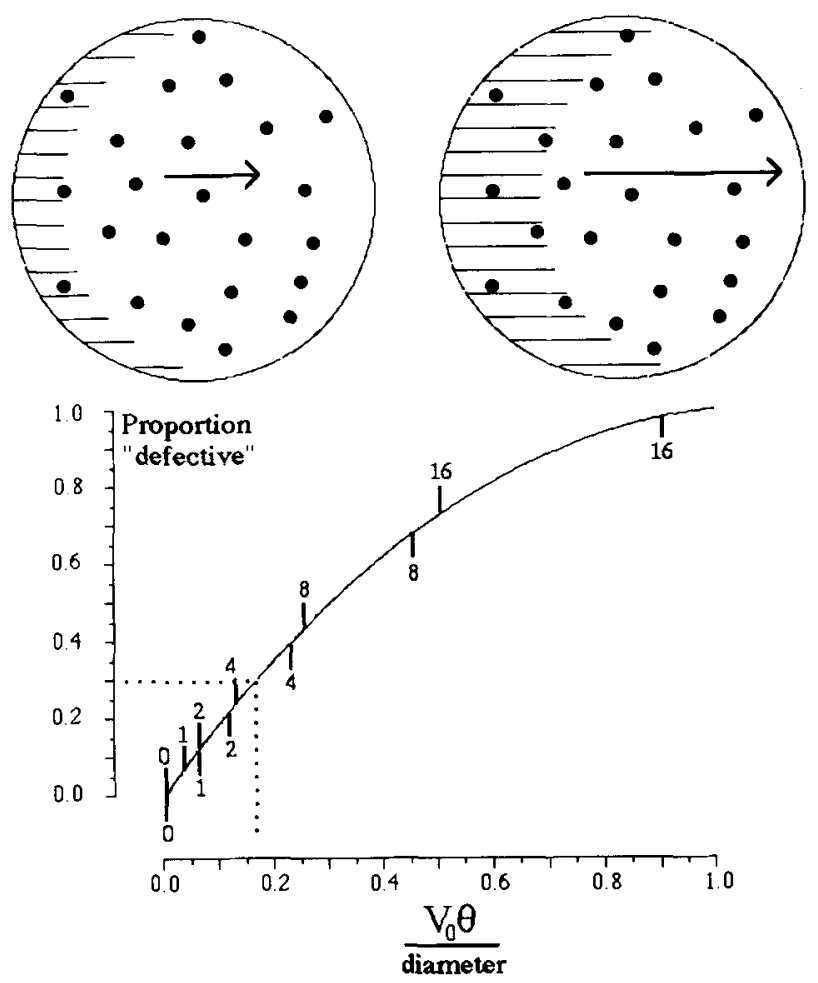

Figure 10. Proportion of dots associated with "defective" adjustments of $\tilde{\mathbf{V}}_{0}$ as a function of distance traversed within the period of adjustment $\theta$ (set equal to $0.5 \mathrm{sec}$ ) in circular display diameter units. The theoretical curve is $(\alpha-\sin \alpha) / \pi+(\pi-\alpha) / \pi \cdot 2 V_{0} \theta / \mathrm{di}-$ ameter, where $\alpha=2 \sin ^{-1}\left(V_{0} \theta /\right.$ diameter $)$. In the diagrams, the area of "defective" adjustments is indicated by horizontal shading for two values of $V_{0}: 0.25$ and 0.5 diameter units/sec. Vertical bars indicate the points on the theoretical curve corresponding to different $V_{0}$ values, shown numerically in degrees per second, for a $16^{\circ}$-diam display (above the curve) and an $8.9^{\circ}$-diam display (below the curve). Pointed lines show the hypothetical distance from the left border within which the dots are being ignored and the corresponding proportion of the ignored dots. 
tions across many dots. Thus, in Figures 8 and 9 the time-position function is presented by a single line, rather than a set of parallel lines for separate coherently moving dots. Our model has been based on the idealized picture in which all computations are essentially identical for all moving dots, so that any reasonable pooling algorithm, at whatever computational stage it takes place, would produce the same result.

Obviously, if the computations are essentially identical for most rather than all moving dots, an effective pooling algorithm that ignores the "outliers" can be easily constructed (e.g., by ignoring the dots that are within a certain critical distance from their starting positions). It is also obvious, however, that the effectiveness of such an algorithm should diminish as the proportion of the "outliers" increases, as it does with increasing $V_{0}$ : Even if all the "outliers" continue to be effectively detected (which seems very difficult, if not impossible), the level of variability in the resultant pooled computation should increase, simply due to the shrinking proportion of the remaining dots. This adds yet another source of noise to those considered in the Appendix for kinematic power $E(t)$. This additional noise, which is dependent on $V_{0}$, makes it necessary to adopt higher values of the parameter $c$ for higher initial velocities. Note that this conclusion is not based on specific assumptions about the pooling algorithm, including the question of whether the pooling occurs at the weight-assignment stage or after the kinematic power has been computed for individual dots.

As an illustration only, in Figure 10 the critical distance from the left border is set at about 0.17 diameter lengths. This value has been chosen so that for a $16^{\circ}$-diam display

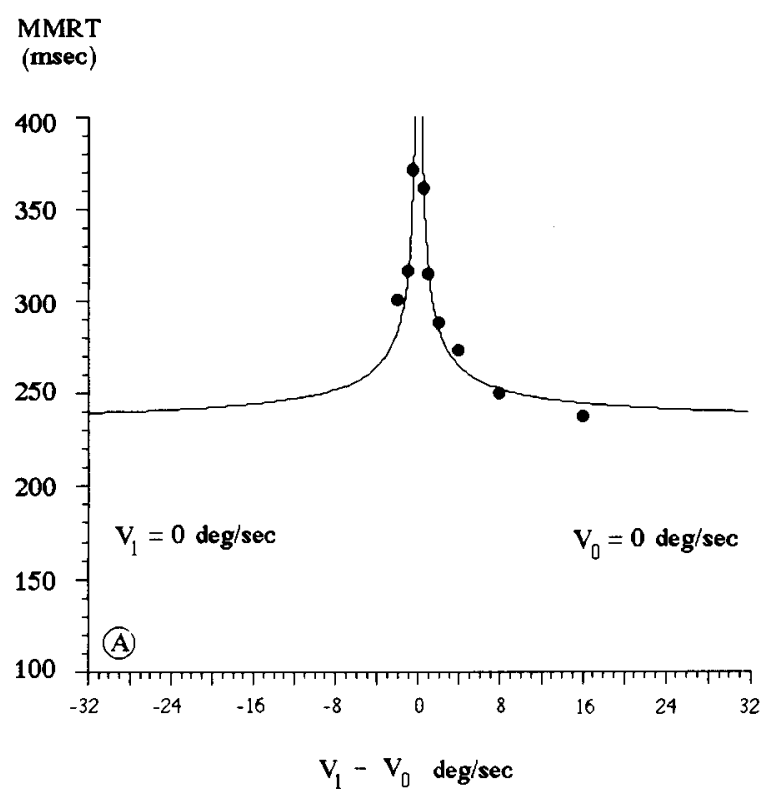

(as in our experiments), assuming $\theta \approx 0.5 \mathrm{sec}$, the critical value of $V_{0}$ falls between 4 and 8 dps. Alternatively, the demarcation line can be thought of as reflecting the critical proportion of ignored dots, beyond which the relative effectiveness of pooled computations decreases. In Figure 10 , this proportion has been set at about $30 \%$. For smaller displays than ours, assuming the same value of $\theta$ and the same demarcation line, the critical value of $V_{0}$ will be lower. Thus, the speed of $4 \mathrm{dps}$ in an $8.9^{\circ}$-diam display corresponds to roughly the same noise level (percent "defective") as $8 \mathrm{dps}$ does in a $16^{\circ}$-diam display. The $8.9^{\circ}$-diam display has been chosen for the comparison because such a display was used in the recent work by Hohnsbein and Mateeff (1992), which we consider next.

Hohnsbein and Mateeff (1992) measured RT to velocity changes in the movement of a random-dot field, $V_{0} t \rightarrow V_{1} t$, with one of the two velocities equal to zero (motion-onset detection, $V_{0}=0$, and motion-offset detection, $V_{1}=0$ ). Their experimental design was similar to ours, with the two most important differences being that (1) as mentioned, they used a smaller aperture $\left(8.9^{\circ}\right.$ in diameter), and (2) their observers maintained steady fixation (the fixation point was located $4.5^{\circ}$ below the aperture's center). Median RTs were arithmetically averaged across observers and plotted against angular speed values in the same range as ours, $0-16 \mathrm{dps}$. Hohnsbein and Mateeff approximated the data by a power function $a+b V^{\gamma}$, where $V$ stands for the nonzero speed in $V_{0} t \rightarrow V_{1} t$, and all three parameters, $a, b$, and $\gamma$, are different for motion onset and motion offset.

Our analysis of these data is shown in Figure 11 and can be summarized as follows. (1) We first fit Equation 7 to

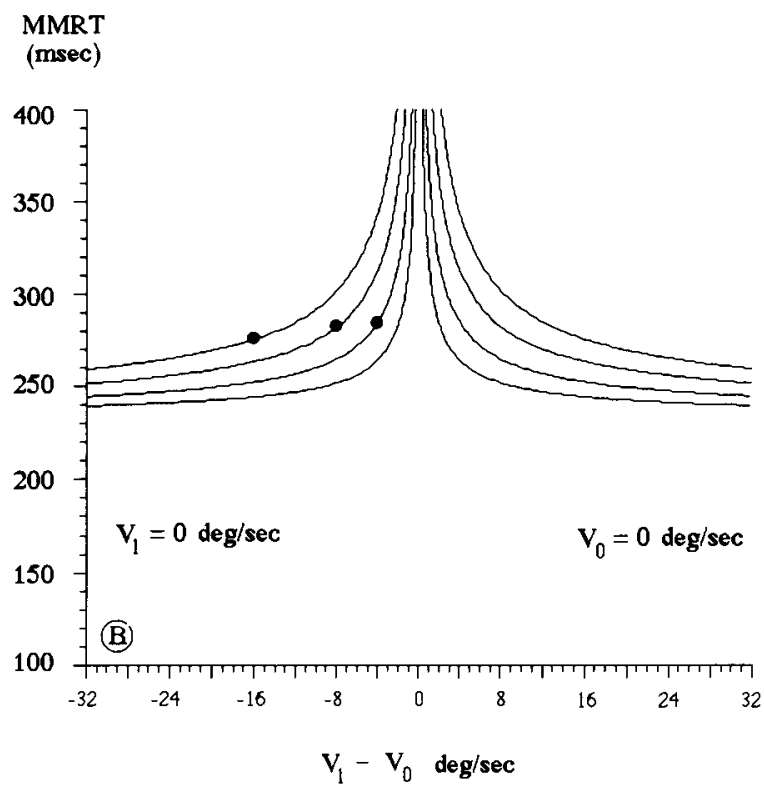

Figure 11. Onset-offset data from Hohnsbein and Mateeff (1992) plotted in the format similar to that of Figures 2 and $3 A$. The vertical axis (MMRT) represents the means computed over median RTs for 6 observers. The curves are of the form $M M R T=r+c\left(V_{0}\right) \mid V_{1}$ $-\left.V_{0}\right|^{-2 / 3}$, where $r=231$ msec, $c(0)=c(0.5)=c(1)=c(2)=0.082, c(4)=0.134, c(8)=0.205, c(16)=0.282\left(\sec ^{1 / 3} \mathrm{deg}^{2 / 3}\right)$. The first of the four curves (for $V_{0}=0,0.5,1,2 \mathrm{dps}$ ) is shown in panel $A$; mean quadratic approximation error is 6.0 msec. This curve is replotted in panel $B$ together with the three curves passing through the points corresponding to $V_{0}=4,8$, and $16 \mathrm{dps}$. 
motion-onset data, (2) then we observe that the points representing $V_{0}=0.5,1$, and 2 dps are reasonably well approximated by the same curve, but that (3) the points representing $V_{0}=4,8$, and $16 \mathrm{dps}$ fall above this curve. According to our model, these three data points lie on three separate curves, corresponding to the same value of $r$ and the same exponent of $-2 / 3$ as for the rest of the data, but to three different values of $c\left(V_{0}\right)$. These curves do not, of course, have any value in testing our model, but are shown only for completeness and comparability with Figures 2 and 3 . The agreement of Figure 11 with these figures is obvious, except for the fact that the critical value of $V_{0}$ is now lower, between 2 and 4 dps. This is, however, precisely what one should expect on the basis of the discussion related to Figure 10.

The similarity between Hohnsbein and Mateeff's (1992) data and our present data is theoretically significant also because it demonstrates that maintaining steady fixation in velocity-change detection does not affect the quantitative form of the relations, as compared with free looking. This fact lends additional support to our assumption that motion encoding, at least to a first approximation, is defined in external rather than retinal coordinates. It also agrees with the results of the experiment with monitored fixation by A. B. Sekuler et al . (1990), discussed in the introductory section of this paper.

Sensory adaptation and readjustment of weights. The readjustment of weights in our model is not necessarily (and probably is not de facto) a sensory adaptation phenomenon, even though the latter can also be described in similar terms. For example, Barlow (1972) discusses sensory adaptation in neuronal networks as a selective amplification of weights related to new information and attenuation of weights related to old information. The weights in this case, however, are understood as a characteristic of the encoding network per se: changes in these weights lead to a transformation of the activation pattern evoked by a given stimulus. Such changes are task nonspecific and manifest themselves in a variety of adaptation aftereffects. In motion perception, these are changes in the perceived velocity of motion (Scott, Jordan, \& Powell, 1963), waterfall illusion (Wohlgemuth, 1911), motion-specific elevation of contrast thresholds (see R. Sekuler, 1975, and Nakayama, 1985, for reviews), and motion-specific elevation of kinematic thresholds (Allik, Tepp, \& Livshits, 1977; Dzhafarov \& Allik, 1981).

We have no evidence that any of these changes took place in our experiments. In fact, for our model to work precisely as intended, we have to assume, at least as an idealization, that the activated encoders are always determined by Equation 8, irrespective of the immediate history of activation. It is important to recognize that the weights in our model are not part of the encoding process per se, but only determine to what extent and which activated units are taken into account in order to answer a particular question about visual motion. In other words, the weights are components of a special-purpose computation performed on the dynamic array of elementary activations encoding the time-position function of a moving target. Many such special-purpose computations can and probably do run in parallel, and each can utilize its own set of weights associated with one and the same dynamic array of elementary activations. The readjustment of weights, therefore, is conceptually closer to the fast learning of Poggio et al. (1992) than to sensory adaptation. We realize, however, that the distinction between the general-purpose encoding and special-purpose detection promoted in this paper may be less than absolute, in which case there might be situations in which transient sensory adaptation would be indistinguishable from task-specific readjustments of summation weights.

Extended principle of the labeled line. The interpretation proposed raises an epistemological question: How does the visual system know which elementary motion encoders should be taken with which weights, and which weights should be transformed to solve the velocitychange-detection task? The answer is: By the labeled line principle. Each motion encoder in our simplified network is characterized by the centers of its two subfields, $X$ and $X+\Delta X$, which thereby become their "labels," fixed values logically assigned to the encoders by the mechanisms receiving their outputs. (If more than one encoder is associated with a given pair of centers, $X$ and $X+\Delta X$, then the labels should be extended to include other fixed characteristics, e.g., sizes of the subfields, sampling phase and frequency, etc.) The mechanisms receiving (in our model, summating) activation of the encoders, therefore, "know" these activations by the encoders' labels, $X$ and $X+\Delta X$. As a result, the manner in which a particular activation is processed (in our model, the weight with which it is pooled with other activations) can be based on the activation label, rather than its value per se (the latter is either 0 or 1 in our model). In detection of velocity changes, the labels of the initial activations (during, say, the first $0.5 \mathrm{sec}$ of the $V_{0}$ phase of the motion) can be used by the detection system to modify the weights with which activations are subsequently summated.

Although widely used, the principle of the labeled line has played a surprisingly limited role in computational models of motion encoding and detection. According to its usual interpretation, all the principle proposes is that visual motion is encoded by specialized mechanisms, so that some motion characteristics, such as direction, are "known" from the labels of the maximally activated mechanisms. That is, from the point of view of a mechanism into which the labeled encoders feed, the labels are on a nominal scale only; the identity of a label can be picked up, or read, but not entered as a value into subsequent computations. It is not difficult to see, however, that the labeled line principle could accommodate labels on scales other than nominal. Quantified labels of the encoding units (such as $X$ and $X+\Delta X$ ) could enter into the visual computations together with the units' variable outputs. This modification of the labeled line principle looks trivial, but it opens new powerful possibilities.

As an abstract example, consider three encoders, $S_{1}, S_{2}$, and $S_{3}$, feeding into another mechanism, $S$. Let the outputs of these encoders received by $S$ be $R_{1}, R_{2}$, and $R_{3}$ (not 
necessarily Boolean, and generally changing in time). Assume that the three encoders have been labeled (from the "point of view" of $S$ ) by three spatial points with coordinates $X_{1}, X_{2}$, and $X_{3}$. The extended principle of the labeled line allows that these coordinates be directly utilized in the computations performed by $S$ together with the outputs $R_{1}, R_{2}, R_{3}$. For example, the response of $S$ can be proportional to $\left|X_{1}-X_{2}\right|$ if the outputs of the three encoders form a certain pattern (say, $R_{1}+R_{2}-R_{3}=1$ ), to $\left|X_{2}-X_{3}\right|$ if they form another pattern, and so on. The computations in our model are, of course, much simpler, but they follow the same general principle: spatial coordinates are not present in the encoders' outputs, but they are built into the circuitry of the detection system $(S)$, and determine its input-output transformations.

\section{REFERENCES}

Allik, J., \& Dzhafarov, E. N. (1984). Reaction time to motion onset: Local dispersion model analysis. Vision Research, 24, 99-101.

Allik, J., TePp, M., \& Livshits, A. (1977). Detection of temporal phase by directionally sensitive units in the human visual system. Acta et Commentationes Universitatis Tartuentis, 429, 107-120.

BALL, K., \& SEKULER, R. (1980). Models of stimulus uncertainty in motion perception. Psychological Review, 87, 435-469.

BarLow, H. B. (1972). A neuron doctrine for perceptual psychology. Perception, 1, 371-394.

BONNET, C. (1977). Visual motion detection models: Features and frequency filters. Perception, 6, 491-500.

BonNET, C. (1982). Thresholds of motion perception. In A. H. Wertheim, W. A. Wagenaar, \& H. W. Leibowitz (Eds.), Tutorials on motion perception (pp. 41-79). New York: Plenum.

Burr, D. C. (1980). Motion smear. Nature, 284, 164-165.

BURR, D. C. (1981). Temporal summation of moving images by the human visual system. Proceedings of the Royal Society of London: Series $B, 211,321-339$.

Burr, D. C., Ross, J., \& Morrone, M. C. (1986). Seeing objects in motion. Proceedings of the Royal Society of London: Series B, 227, 249-265.

Cohen, R. L., \& Bonnet, C. (1972). Movement detection thresholds and stimulus duration. Perception \& Psychophysics, 12, 269-272.

Collewun, H. (1972). Latency and gain in the rabbit's optokinetic reactions to small movements. Brain Research, 36, 59-70.

Dzhafarov, E. N. (1992). The structure of simple reaction time to stepfunction signals. Journal of Mathematical Psychology, 36, 235-268.

Dzhafarov, E. N. (1993). Grice-representability of response time distribution families. Psychometrika, 58, 281-314.

Dzhafarov, E. N., \& Allik, J. (1981). Selective visual adaptation and the visual feature model. In Y. M. Zabrodin (Ed.), Psychological studies in perception and memory (pp. 77-92). Moscow: Nauka.

Dzhafarov, E. N., \& Allik, J. (1984). A general theory of motion detection. In M. Rauk (Ed.), Computational models in hearing and vision (pp. 77-84). Tallinn: Estonian Academy of Sciences.

Dzhafarov, E. N., Allik, J., \& Linde, N. D. (1983). Detection of oscillatory movement. Voprosy Psikhologii, 3, 90-96.

Dzhafarov, E. N., Allik, J., Linde, N. D., \& Piastolov, V. K. (1981). Comparative analysis of frequency-amplitude threshold functions for real and apparent motion. Psikhologicheskii Zhumal, 2, 73-78.

FAHLE, M., \& POGGIO, T. (1981). Visual hyperacuity: Spatio-temporal interpolation in human vision. Proceedings of the Royal Society of London: Series B, 213, 451-477.

Hallett, P. E. (1986). Eye movements. In K. R. Boff, L. Kaufman, \& J. P. Thomas (Eds.), Handbook of perception and human performance (Vol. 1, pp. 10.1-10.112). New York: Wiley.

Hohnsbein, J., \& MateefF, S. (1992). The relation between the velocity of visual motion and the reaction time to motion onset and offset. Vision Research, 32, 1781-1791.
Johnson, C. A., \& Leibowitz, H. W. (1976). Velocity-time reciprocity in the perception of motion: Foveal and peripheral determinations. Vision Research, 16, 177-180.

Johnson, C. A., \& Scobey, R. P. (1980). Foveal and peripheral displacement thresholds as a function of stimulus luminance, line length and duration of movement. Vision Research, 20, 709-715.

Kowler, E. (1990). The role of visual and cognitive processes in the control of eye movement. In E. Kowler (Ed.), Eye movements and their role in visual and cognitive processes ( $\mathrm{pp} .1-70$ ). Amsterdam: Elsevier.

LUCE, R. D. (1986). Response times. New York: Oxford University Press.

Mitrani, L., \& Dimitrov, G. (1978). Pursuit eye movements of a disappearing moving target. Vision Research, 18, 537-540.

Mitrani, L., Dimitrov, G., Yakimoff, N., \& MateefF, S. (1979). Oculomotor and perceptual localization during smooth eye movements. Vision Research, 19, 609-612.

NAKAYAmA, K. (1985). Biological image motion processing: A review. Vision Research, 25, 625-660.

Nakayama, K., \& TYler, C. W. (1978). Relative motion induced between stationary lines. Vision Research, 18, 1663-1668.

Poggio, T., Fahle, M., \& Edelman, S. (1992). Fast perceptual learning in visual hyperacuity. Science, 256, 1018-1021.

RATCLIFF, R. (1979). Group reaction time distributions and an analysis of distribution statistics. Psychological Bulletin, 86, 446-461.

REICHARDT, W. (1961). Autocorrelation, a principle for the evaluation of sensory information by the central nervous system. In W. A. Rosenblith (Ed.), Sensory communications (pp. 303-317). Cambridge, MA: M.I.T. Press.

SCOBEY, R. P., \& Johnson, C. A. (1981). Displacement thresholds for unidirectional and oscillatory movement. Vision Research, 21, 1297-1302.

Scott, T. R., Jordan, A. E., \& Powell, D. A. (1963). Does the visual aftereffect of motion add algebraically to objective motion of the test stimulus? Journal of Experimental Psychology, 66, 500-505.

Sekuler, A. B., Sekuler, E. B., \& Sekuler, R. (1990). How the visual system detects changes in the direction of moving targets. Perception, 19, 181-196.

SeKUler, R. (1975). Visual motion perception. In E. C. Carterette \& M. P. Friedman (Eds.), Handbook of perception (Vol. 5, pp. 387430). New York: Academic Press.

Troscianko, T., \& FAHLE, M. (1988). Why do isoluminant stimuli appear slower? Journal of the Optical Society of America A, 5, 871-879.

TYLER, C. W., \& TORRES, J. (1972). Frequency response characteristics for sinusoidal movement in the fovea and periphery. Perception \& Psychophysics, 12, 232-236.

Tynan, P. D., \& Sekuler, R. (1982). Motion processing in peripheral vision: Reaction time and perceived velocity. Vision Research, 22, 61-68.

VAN DEN BERG, A. V., \& VAN DE GRIND, W. A. (1989). Reaction times to motion onset and motion detection thresholds reflect the properties of bilocal motion detectors. Vision Research, 29, 1261-1266.

VAN DE GRIND, W. A., KoENDERINK, J. J., \& VAN DoORN, A. J. (1983). Detection of coherent movement in peripherally viewed random-dot patterns. Journal of the Optical Society of America, 73, 1674-1683.

van DE Grind, W. A., Koenderink, J. J., \& VAN Doorn, A. J. (1986). The distribution of human motion detector properties in the monocular visual field. Vision Research, 26, 797-810.

van Doorn, A. J., \& KoEnderink, J. J. (1982a). Spatial properties of the visual detectability of moving spatial white noise. Experimental Brain Research, 45, 189-195.

van DoORn, A. J., \& Koenderink, J. J. (1982b). Temporal properties of the visual detectability of moving spatial white noise. Experimental Brain Research, 45, 179-188.

VAN DoORn, A. J., \& KoEnderink, J. J. (1983). The structure of the human motion detection system. IEEE Transactions on Systems, Man, and Cybernetics, SMC-13, 916-922.

van Doorn, A. J., \& Koenderink, J. J. (1984). Spatiotemporal integration in the detection of coherent motion. Vision Research, 24, 47-53. 
van Doorn, A. J., Koenderink, J. J., \& van de Grind, W. A. (1984) Limits in spatio-temporal correlation and the perception of visual movement. In A. J. van Doorn, J. J. Koenderink, \& W. A. van de Grind (Eds.), Limits in perception (pp. 203-234). Utrecht: VNU Science Press.

van Santen, J. P. H., \& Sperling, G. (1984). Temporal covariance model of human motion perception. Journal of the Optical Society of America A, 1, 451-473

van Santen, J. P. H., \& SPerling, G. (1985). Elaborated Reichardt detectors. Journal of the Optical Society of America A, 2, 300-321.

Westheimer, G., \& McKee, S. P. (1975). Visual acuity in the presence of retinal image motion. Joumal of the Optical Society of America, $65,847-850$

Westhejmer, G., MCKeE, S. P. (1977). Integration regions for visual hyperacuity. Vision Research, 17, 89-93.

Whittaker, S. G., \& Eaholtz, G. (1982). Learning patterns of eye motion for foveal pursuit. Investigative Ophthalmology \& Visual Science, 23, 393-397.

Wohlgemuth, A. (1911). On the after-effect of seen motion. British Journal of Psychology, Monograph Suppl., November 1.

\section{NOTES}

1. We confine ourselves to this descriptive characterization of the goodness of fit because the traditional lack-of-fit $F$ statistics (testing Equation 7 against the hypothesis that all RT distributions are well described by their means) would make very little sense here, for two reasons (aside from the obvious fact that the traditional distributional assumptions, normality and homoscedasticity, are grossly violated in the case of RT distributions). First, as shown in the Discussion sections and the Appendix, the negative-exponent power functions are explicitly introduced as only a first-order approximation to "true" functions. Second, the analysis of the RT distributions in terms of their moments after they have been censored (trimmed) below and above certain values $(0.1$ and $1 \mathrm{sec}$ in our case) is itself a crude procedure that can only be justified if the analysis is being confined to MRTs only (due to their robustness under censoring). The variance values (that are necessary for computing lackof-fit statistics) and higher moments are sensitive to censoring and are therefore unreliable (see Dzhafarov, 1992, for a more detailed discussion of these issues; see also Ratcliff, 1979).

2. This description involves a simplifying assumption, which we suggest may be acceptable at least insofar as rigidly moving sharply localized patterns are concerned. The assumption is that the luminance profile characteristics are sampled by an encoder at certain moments (i.e., intervals whose duration is negligibly small) rather than within extended intervals, so that the luminance profiles are only characterized by their spatial rather than spatiotemporal properties. Indirect evidence that this simplification may in fact be plausible can be found in the series of observations that the visual system within a certain range of velocities and exposure times can effectively preserve spatial details of a moving pattern (Burr, 1980, 1981; Burr, Ross, \& Morrone, 1986; Fahle \& Poggio, 1981; Westheimer \& McKee, 1975, 1977).

3. The original version of the model was termed the "local dispersion model," and it contained one additional operation: smoothing of $E(t)$ by averaging its values across a large time window. Some form of smoothing may indeed be a plausible assumption when dealing with justvisible motion thresholds measured by the method of adjustments. Indeed, if $E(t)$ reflexes the perceptual salience of motion, then the observer is compelled to adjust motion parameters so that $E(t)$ is at some criterion value on average. In the $\mathrm{RT}$ paradigm, however, the observer simply responds as soon as the criterion value is reached for the first time. The averaging assumption in this case is not justified, contrary to Allik and Dzhafarov (1984).

\section{APPENDIX}

In this appendix we derive the approximate formulas for MRT to onset of uniform motion, $0 \rightarrow V t$, and to instantaneous shift in the target position: $0 \rightarrow A$. We also briefly consider the effect of additive and multiplicative computational errors on the detection algorithm.

Equation 13 is equivalent to

$$
\begin{aligned}
E(t) & =\frac{1}{2} \tau^{-2} \int_{t-\tau}^{t} \int_{t-\tau}^{t}\left[x\left(t_{2}\right)-x\left(t_{1}\right)\right]^{2} d t_{2} d t_{1} \\
& =\tau^{-1} \int_{t-\tau}^{t}[x(u)]^{2} d u-\left[\tau^{-1} \int_{t-\tau}^{t} x(u) d u\right]^{2} .
\end{aligned}
$$

According to the detection rule adopted in this paper, motion detection occurs when $E(t)$ reaches a criterion $C$ for the first time ( $C$ is a nonnegative random variable). If an additive localization error $\mathbf{E}$ is involved, so that $x(u)$ in Equation $\mathrm{Al}$ should be replaced with $x(u)+\mathbf{E}$, then, assuming that $\mathbf{E}$ is independent of $x$ and its autocorrelation in time is close to a delta function, Equation Al transforms into

$$
E(t)=E(t)_{\text {ideal }}+\sigma^{2}(\mathbf{E}),
$$

where $\sigma^{2}(\mathbf{E})$ is the variance of $\mathbf{E}$, and $E(t)_{\text {ideal }}$ is the value computed according to Equation Al. The detection rule $E(t)=\mathbf{C}$ can now be rewritten as $E(t)_{\text {ideal }}=\mathbf{C}^{*}-\sigma^{2}(\mathbf{E})$, and to prevent false alarms, the minimum of $\mathbf{C}^{*}$ should be adjusted to exceed $\sigma^{2}(\mathbf{E})$. Since thus defined $\mathbf{C}^{*}-\sigma^{2}(\mathbf{E})$ is a nonnegative random variable, it can be renamed back to $\mathbf{C}$, thereby restoring the original (error-free) formulation of the detection rule: $E(t)_{\text {ideal }}=\mathbf{C}$. (The approximate delta-function assumption is only a mathematical simplification-it can be dropped without affecting the final computational formulas. The independence assumption is more important: the model becomes much more flexible if $\mathbf{E}$ is allowed to correlate with $x$.) A multiplicative error is introduced and handled analogously. If spatial shifts $\left[x\left(t_{2}\right)-x\left(t_{1}\right)\right]$ are matched to spatial spans $\Delta X$ with a positive multiplicative error $\mathrm{E}_{\mathrm{m}}$, then $\left[x\left(t_{2}\right)-x\left(t_{1}\right)\right]$ in Equation $\mathrm{A} 1$ should be replaced with $\mathbf{E}_{\mathrm{m}}\left[x\left(t_{2}\right)-x\left(t_{1}\right)\right]$. Under assumptions analogous to those made above, the situation is reduced to the following redefinition of the detection rule:

$$
E(t)_{\text {ideal }}=\mathbf{C}=\frac{\mathbf{C}^{*}-\sigma^{2}(\mathbf{E})}{\mu\left(\mathbf{E}_{m}^{2}\right)},
$$

where $\mu$ stands for the mean value.

Applying Equation Al to the $0 \rightarrow V t$ stimulus,

$$
x(t)=\left\{\begin{array}{l}
0 \text { if } t \leq 0 \\
V t \text { if } t>0
\end{array},\right.
$$

we have

$$
E(t)=\left\{\begin{array}{cc}
(V \tau)^{2}\left[\frac{1}{3}\left(\frac{t}{\tau}\right)^{3}-\frac{1}{4}\left(\frac{t}{\tau}\right)^{4}\right] & \text { if } t<\tau \\
\frac{1}{12}(V \tau)^{2} & \text { if } t \geq \tau
\end{array}\right\}
$$

Equating Equation $\mathrm{A} 2$ to $\mathbf{C}$ and solving for $t$, one gets an expression for the velocity-dependent component $D(V, \mathbf{C})$ of $\mathbf{R T}$ (Equation 2). Assuming that $t$ is very small compared with $\tau$, we can omit in Equation $\mathrm{A} 2$ all powers of $t / \tau$ but the lowest, which yields 


$$
D(V, \mathbf{C}) \approx(3 \mathrm{C} \tau)^{1 / 3} V^{-2 / 3} .
$$

Consequently,

$$
\operatorname{MRT}(V) \approx c V^{-2 / 3}+r,
$$

where $c$ is the mean value of $(3 \mathbf{C} \tau)^{1 / 3}$, and $r$ is the mean value of $\mathbf{R}$, the velocity-independent component of $\mathbf{R T}$.

Now applying Equation $\mathrm{A} 1$ to the $0 \rightarrow A$ stimulus,

$$
x(t)=\left\{\begin{array}{l}
0 \text { if } t \leq 0 \\
A \text { if } t>0
\end{array},\right.
$$

we have

$$
E(t)=\left\{\begin{array}{cc}
A^{2}\left[\left(\frac{t}{\tau}\right)-\left(\frac{t}{\tau}\right)^{2}\right] & \text { if } t<\tau \\
0 & \text { if } t \geq \tau
\end{array}\right\},
$$

where the second power of $t / \tau$ can be dropped if one assumes $t / \tau \ll 1$. Equating Equation A4 to $\mathbf{C}$ and solving for $t$, one gets the following approximate expressions for the amplitudedependent component $D(A, \mathrm{C})$ of $\mathrm{RT}$ :

$$
D(A, \mathbf{C}) \approx(\mathbf{C} \tau) A^{-2} .
$$

Consequently,

$$
\operatorname{MRT}(A) \approx c A^{-2}+r,
$$

where $c$ now is the mean value of $\left(C_{\tau}\right)$, and $r$ is the mean value of $\mathbf{R}$, interpreted as the amplitude-independent component of $\mathbf{R T}$.

It is of great importance that the inverse squaring of amplitude in Equation A5 and squaring of shifts in Equation A1 are interdeducible. If one replaces the squaring operation in Equation A1 with some other function $w\left(\left|x\left(t_{2}\right)-x\left(t_{1}\right)\right|\right)$, then Equation A5 assumes the form

$$
D(A, \mathbf{C}) \approx(\mathbf{C} \tau) w(A)^{-1} .
$$

As a result, the operation $w\left(\left|x\left(t_{2}\right)-x\left(t_{1}\right)\right|\right)$, that is, the weight assigned to encoders with the spatial span of $\left|x\left(t_{2}\right)-x\left(t_{1}\right)\right|$, can be empirically identified as squaring by showing that MRT is indeed a linear function of $A^{-2}$ (for a detailed discussion of linearizing transformations, see Dzhafarov, 1992).

(Manuscript received November 20, 1992; revision accepted for publication April 30, 1993.) 